\title{
Anomaly Detection for Mobile Device Comfort
}

by

\section{Mehmet Vefa Bicakci}

\author{
A Thesis submitted to \\ the Faculty of Graduate Studies and Research \\ in partial fulfilment of \\ the requirements for the degree of \\ Master of Applied Science \\ Ottawa-Carleton Institute for \\ Electrical and Computer Engineering
}

Department of Systems and Computer Engineering

Carleton University

Ottawa, Ontario, Canada

August 2013

Copyright (C)

2013 - Mehmet Vefa Bicakci 
The undersigned recommend to

the Faculty of Graduate Studies and Research

acceptance of the Thesis

\title{
Anomaly Detection for Mobile Device Comfort
}

\author{
Submitted by Mehmet Vefa Bicakci \\ in partial fulfilment of the requirements for the degree of \\ Master of Applied Science
}

(a)




\section{Abstract}

In this work, we utilize the data that can be collected via some of the sensors found on modern smartphones to find anomalies in the behaviour of a smartphone user and the current context. This can be done to prevent the mobile phone from being compromised physically or to warn the user when he/she is behaving "unusually."

We apply the "time slice" notion to existing anomaly detection methods, evaluate our approach on two published data sets, and confirm that it is feasible to use our approach on smartphones with modest hardware.

Our work is part of Marsh et al.'s Device Comfort paradigm, which is an application of computational trust to mobile device security, where the mobile device is able to have a varying level of "trust" in its user based on behavioural biometrics, and the contexts in which the user interacts with the mobile device. 


\section{Acknowledgements}

First of all, I would like to thank Professor Esfandiari and Professor Marsh for being very understanding and helpful, and for guiding me through the thesis process.

Thanks to Alan Davoust for the conceptual discussions regarding this thesis, and for the tea! Thanks to Michael Floyd for being helpful as well, especially during my second semester.

Finally, I would like to thank my family for their encouragement and support during the thesis process. 


\section{Table of Contents}

Abstract $\quad$ iii

Acknowledgements $\quad$ iv

Table of Contents $\quad$ v

List of Tables $\quad$ ix

List of Figures $\quad$ X

Nomenclature $\quad$ xiii

1 Introduction 1

1.1 Device Comfort . . . . . . . . . . . . . . . 2

1.2 Research Problem . . . . . . . . . . . . . . . . . 3

1.3 Overview of Our Work . . . . . . . . . . . . . . 4

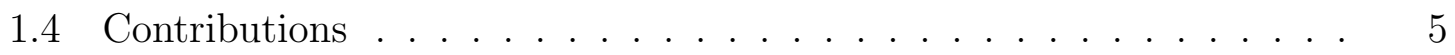

1.5 Thesis Structure . . . . . . . . . . . . . . . 6

2 Background $\quad 8$

2.1 Types of Anomaly Detection Approaches . . . . . . . . . . . 8

2.2 Two Anomaly Detection Algorithms . . . . . . . . . . . . 9

2.2.1 Fixed-Width Clustering Algorithm . . . . . . . . . . . . 10 
2.2.2 An Optimized k-Nearest Neighbours Algorithm . . . . . . . . 11

2.2.3 Discussion . . . . . . . . . . . . . . . . . . . . . . 13

2.3 Performance Metrics in Machine Learning . . . . . . . . . . . . . . . 13

2.4 Fusion Methods in Biometrics . . . . . . . . . . . . . . . . . . 17

2.5 Conclusion . . . . . . . . . . . . . . . . . . . . 18

3 Related Work $\quad 20$

3.1 Introduction . . . . . . . . . . . . . . . . . . . . 21

3.2 Targeted Platforms and Implementation Status _ . . . . . . . . . 22

3.3 Features . . . . . . . . . . . . . . . . . . . . 24

3.4 Methodologies ..................... 26

3.4.1 Branscomb .................... 27

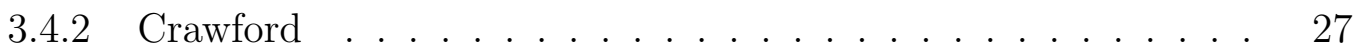

3.4.3 Li et al. . . . . . . . . . . . . . . . . . . 28

3.4.4 Salem et al. . . . . . . . . . . . . . . . . . . . . . 29

3.4.5 Shi et al. . . . . . . . . . . . . . . . . . 29

3.4.6 Yazji et al.'s Spatio-Temporal Anomaly Detection Proposal . . 30

3.4.7 Yazji et al.'s File-System and Network Access-based Anomaly Detection Proposal . . . . . . . . . . . . . . . . . 30

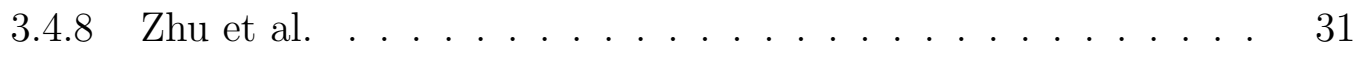

3.5 Data Sets . . . . . . . . . . . . . . . . . . . 32

3.6 Evaluation . . . . . . . . . . . . . . . . . . . 34

3.6.1 Salem et al. . . . . . . . . . . . . . . . 35

3.6.2 Yazji et al.'s File-System and Network Access-based Anomaly Detection Proposal . . . . . . . . . . . . . 35

3.6.3 Zhu et al. . . . . . . . . . . . . . . . 36

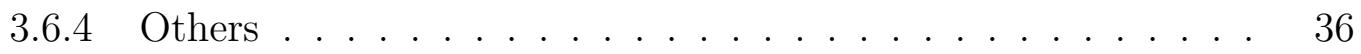


3.7 Performance Metrics . . . . . . . . . . . . . . . . . . . . . . 37

3.8 Conclusion . . . . . . . . . . . . . . . . . . . . . . 37

4 Methodology $\quad 41$

4.1 Data Sets . . . . . . . . . . . . . . . . . . 41

4.2 Data Set Limitations . . . . . . . . . . . . . . . . . . . . . 42

4.3 Overview of the Features Available in the Data Sets . . . . . . . . . 43

4.3.1 Reality Mining Data Set . . . . . . . . . . . . . 43

4.3.2 Social Evolution Data Set . . . . . . . . . . . . . . . 44

4.4 Feature Selection . . . . . . . . . . . . . . . 45

4.4.1 Reality Mining Data Set . . . . . . . . . . . . 45

4.4.2 Social Evolution Data Set . . . . . . . . . . . . . . 46

4.5 Preprocessing of the Reality Mining Data Set . . . . . . . . . . 46

4.6 Data Model . . . . . . . . . . . . . . . . . 47

4.6.1 Regarding "Empty" Time Slices . . . . . . . . . . . . . . . . . 49

4.7 Distance Function . . . . . . . . . . . . . . . . . . . . . 50

4.8 Fusion .............................. 54

4.8.1 Motivation for Match Score Level Fusion . . . . . . . . . . . . 54

4.8.2 Match Score Level Fusion Methodology . . . . . . . . . . . . . 55

4.9 Conclusion . . . . . . . . . . . . . . . . 56

5 Evaluation $\quad 57$

5.1 Dynamic User Profiles . . . . . . . . . . . . . . . . . . 58

5.2 Multi-Stage Cluster Sampling . . . . . . . . . . . . . 58

5.3 Important Notes . . . . . . . . . . . . . . . . . 59

5.4 Effect of Multi-Stage Cluster Sampling on Performance . . . . . . . . 60

5.5 Effect of Classifier Parameters on Performance . . . . . . . . . 63

5.6 Classification Performance of Individual Features . . . . . . . . . 65 
5.7 Classification Performance with Different Training Period Lengths . . 70

5.8 Classification Performance with Different Time Slice Lengths . . . . . 71

5.9 Results with Match Score Level Fusion . . . . . . . . . . . . . . . . 73

5.10 Comparison to a Method without Time Slices . . . . . . . . . . . . 76

5.10.1 Overview of the Method without Time Slices . . . . . . . . 76

5.10 .2 Evaluation Overview . . . . . . . . . . . . . . . 78

5.10 .3 Results........................ . . 78

5.10 .4 Limitations . . . . . . . . . . . . . . . . . . 79

5.11 Comparison of Our Results with Those of $\mathrm{Li}$. . . . . . . . . . . 80

5.12 Conclusion . . . . . . . . . . . . . . . . . . . 82

6 Deployment $\quad 84$

6.1 Target Mobile Device Platform _. . . . . . . . . . . . . 84

6.2 Experimental Set-up . . . . . . . . . . . . . . 86

6.2.1 Collected Features _. . . . . . . . . . . . . . 86

6.2.2 Data Collection and Anomaly Detection . . . . . . . . . . 87

6.2.3 Description of Experiment . . . . . . . . . . . . . . 88

6.3 Results and Discussion . . . . . . . . . . . . . . . . . . . . . . . . 88

6.4 Conclusion . . . . . . . . . . . . . . . . . . . . . . . . 90

$\begin{array}{llr}7 & \text { Conclusion } & 91\end{array}$

7.1 Limitations and Future Work _. . . . . . . . . . . . . . . . . 92

7.2 Conclusion . . . . . . . . . . . . . . . . . . . 95

$\begin{array}{ll}\text { List of References } & 96\end{array}$ 


\section{List of Tables}

2.1 Confusion Matrix . . . . . . . . . . . . . . . . . . 14

3.1 Summary of Related Work (Part 1 of 2 ) . . . . . . . . . . . . 39

3.2 Summary of Related Work (Part 2 of 2) . . . . . . . . . . . . . . 40

5.1 Li's Results in Terms of the EER Metric ${ }^{2} \ldots$. . . . . . . . . . . 81

6.1 Comparison of the Capabilities of Our Target Smartphone to Those of a Recent Smartphone . . . . . . . . . . . . . . . . . . . . . 85 


\section{List of Figures}

2.1 A Fictitious Plot of FPR and FNR against Threshold ${ }^{2} \ldots$. . . . . 16

2.2 A Receiver Operating Characteristic (ROC) Curve . . . . . . . . . 17

4.1 Two examples of time slice data structures. . . . . . . . . . . . . . . . 49

5.1 Illustration of a Dynamic User Profile Sub-experiment. In Figure 5.1a, a classifier is trained on the first $N$ days' data and tested with the $(N+1)$ th day's data and a sample of the attacker participants' data. For the second iteration, which is illustrated in Figure 5.1b, we shift the training period and the negative testing day to the right by one day, and take a new sample of positive time slices from the attacker participants' data. . . . . . . . . . . . . . . . . 59

5.2 Comparison of the Experiment Results with and without Multi-Stage Cluster Sampling . . . . . . . . . . . . . . . .

5.3 Comparison of the Experiment Results with and without Multi-Stage Cluster Sampling . . . . . . . . . . . . . . . . . .

5.4 Comparison of the Experiment Results with and without Aggressive Multi-Stage Cluster Sampling . . . . . . . . . . . . .

5.5 Effects of Classifier Parameters on Performance (AUC and EER) Shown with Boxplots (cw Refers to Fixed-Width Clustering Algorithm's Cluster Width Parameter, and $\mathrm{k}$ Refers to the $k$ in k-NN.) . 
5.6 k-NN Classification Performance (AUC and EER) of Different Features of the Reality Mining Data Set (k is 1, time slice length is 4 hours, "leniency" is 4 hours, training period length is 21 days) . . . . . . .

5.7 k-NN Classification Performance (AUC and EER) of Different Features of the Social Evolution Data Set (k is 1, time slice length is 4 hours, "leniency" is 4 hours, training period length is 21 days) . . . . . . . 68

5.8 Scatter Plots of the AUC Metric Showing the Variance of the Metric between Iterations . . . . . . . . . . . . . . . . . . . . 70

5.9 k-NN Classification Performance (AUC and EER) of Different Training Period Lengths with the Reality Mining Data Set (All features, k is 1, time slice length is 4 hours, "leniency" is 4 hours) . . . . . . . . . . 72

5.10 k-NN Classification Performance (AUC and EER) of Different Training Period Lengths with the Social Evolution Data Set (All features, k is 1 , time slice length is 4 hours, "leniency" is 4 hours) . . . . . . . .

5.11 k-NN Classification Performance (AUC and EER) of Different Time Slice Lengths with the Reality Mining Data Set $(R M)$ and the Social Evolution Data Set $(S E)$ (Aggressive sampling, all features, k is 1, "leniency" is 4 hours, training period length is 21 days) . . . . . . . . 74

5.12 Boxplots Depicting a Pairwise Comparison of the k-NN Classification Performance (AUC) per Iteration (or Test Day). (Aggressive sampling, all features, $\mathrm{k}$ is 1 , "leniency" is 4 hours, training period length is 21 days $\ldots \ldots \ldots \ldots \ldots \ldots \ldots$

5.13 AUC and EER Results of Different Fusion Methods with k-NN and the Reality Mining Data Set $(R M)$ and the Social Evolution Data Set $(S E)$ (All features, $\mathrm{k}$ is 1 , time slice length is 4 hours, "leniency" is 4 hours, training period length is 21 days) . . . . . . . . . . 
5.14 Boxplot Depicting a Pairwise Comparison of Classification Performance (AUC) per Iteration (or Test Day) of Match Score Level Voting and Feature Level Fusion with the Reality Mining Data Set. (All features, $\mathrm{k}$ is 1 , time slice length is 4 hours, training period length is 21 days) . . . . . . . . . . . . . . . . . . . . 76

5.15 AUC and EER Results of the Two Rule-based Approaches and the 1-Hour-long Time Slice-based Approach with the Reality Mining Data

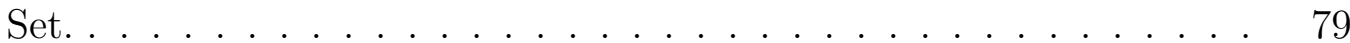

5.16 Boxplots Depicting a Pairwise Comparison of Classification Performance (AUC and EER) per Iteration of 1-Hour-long Time Slice-based Method and Rule-based Method with Location Feature Fusion (on the Reality Mining Data Set). . . . . . . . . . . . . . 


\title{
Nomenclature
}

\author{
AUC Area Under the (ROC) Curve \\ BWC Binary Weighted Cosine (Metric) \\ EER Equal Error Rate \\ FN False Negative \\ FNR False Negative Rate \\ FP False Positive \\ FPR False Positive Rate \\ GPS Global Positioning System \\ IDS Intrusion Detection System \\ k-NN k-Nearest Neighbours \\ MAC Media Access Control (Address) \\ ROC Receiver Operating Characteristic (Curve) \\ SVM Support Vector Machine \\ TNR True Negative Rate \\ TN True Negative \\ TPR True Positive Rate \\ TP True Positive
}




\section{Chapter 1}

\section{Introduction}

Modern smartphones have become convergent platforms that can be used for browsing the web, making online payments, taking photographs, geo-location and, of course, placing phone calls. Furthermore, one can expect to find many sensors on recent mid-end to high-end mobile phones, including accelerometers, gyroscopes, compasses, gravity sensors, light and proximity sensors, touch-screens, cameras, Global Positioning System (GPS) sensors, and Bluetooth, Wi-Fi and cellular capabilities.

As mobile phones become more convergent, the data that we store on them becomes larger in quantity and more valuable. A quick search on Google Play ${ }^{1}$ for Canadian banking institutions returns the names of highly reputable banks, the applications of which are downloaded on the order of 100, 000 times in one month. (As of 6 May 2013.)

Banking details (which are important enough on their own) may not be the only kind of sensitive information we handle using a modern smartphone, which may contain personal photographs, important e-mails, web browsing history and authentication details for web sites.

Given the increasing importance of the mobile convergent platforms, the wide variety of tasks for which they are used, and the sensitivity of the information they

\footnotetext{
${ }^{1}$ Google Play is the application market for Google's Android operating system for mobile devices.
} 
contain, there is an emerging need to protect the information stored on such devices.

In this document, we utilize the sensor data that can be collected via some of the above-mentioned sensors of modern smartphones to find anomalies in the behaviour of the user of a mobile phone and the current context. This can be done to prevent the mobile phone from being compromised physically or to warn the user when he/she is behaving "unusually," with regards to the overall behavioural patterns that the user has established with his/her mobile phone, which is itself useful information.

\subsection{Device Comfort}

This work contributes to the overall architecture of the "Device Comfort" concept, as described by Marsh et al. in [1]. Device Comfort is an application of computational trust to mobile personal computing device security, where the mobile device - such as a smartphone - in an anthropomorphic way, is able to have a varying level of trust in its user based on behavioural biometrics, and the contexts in which the interactions between the personal computing device and its user take place. Policies set up by the owner, who may or may not be the same entity as the current user, of a personal computing device are also influential in Device Comfort.

As part of trusting its user, a comfort-enabled personal computing device utilizes behavioural biometrics, ${ }^{2}$ examples of which include keystroke, voice and gait dynamics, and patterns in Human-Computer Interaction, such as application usage patterns, and patterns in file-system and network accesses made by the user.

The contextual information used by a comfort-enabled personal computing device includes the current task, location, and social setting of the device.

Device Comfort is related to authentication, access control, and confidentiality,

\footnotetext{
${ }^{2}$ According to [2], behavioural biometrics is based "on skills, style, preference, knowledge, motorskills or strategy used by people while accomplishing different everyday tasks such as [...] using a computer."
} 
where, based on the available behavioural biometric and contextual information, the comfort-enabled device, based on its level of contextually-determined security posture, can let the user authenticate himself/herself to the device in order to access data related to the current context (or conversely, disallow access to information unrelated to the current context), which may be decrypted with keys stored on the device itself, or on other nearby comfort-enabled wireless devices.

Contextual information pertaining to the current location of the comfort-enabled personal computing device can be modelled with "Comfort Zones" and "Discom-

fort Zones," [1] which are zones (as inferred via GPS or cellular tower-based spatial information sources) in which the mobile device feels more "comfortable" or "uncomfortable" and would allow or disallow, respectively, the user to perform tasks or access data that require a certain level of trust in the user and the current context.

"Social Zones," on the other hand, involve the (comfort-enabled devices of) people related to the owner or the user of the mobile device in question. [1] For example, after sensing the mobile devices of a group of business colleagues, a user's mobile device may allow access to files containing information related to business, which would not be the case if the detected mobile devices belonged to close friends.

Finally, policies in a comfort-enabled mobile device can be used to allow or proscribe specific personal computing related behaviours facilitated by the mobile device, based on the context. These policies may be set by the owner of the mobile device, who may not be its current user - for example, one of the parents of a teenage user, or the employer of an enterprise employee.

\subsection{Research Problem}

Performing anomaly detection on the behavioural and contextual patterns in the interactions between a mobile personal computing device and its user is one of the 
building blocks of Device Comfort. As such, in this thesis, we focus on the following research problem:

How can a comfort-enabled mobile device detect anomalies in the context and the behavioural patterns of its user?

This research problem can be composed of the following research sub-problems:

1. How can we summarize the contextual and behavioral patterns of a mobile device user, and compare the summarized information to past patterns for anomaly detection purposes?

2. What are the most promising features available in published data sets that can be used for anomaly detection in a comfort-enabled device?

3. Is it feasible to run anomaly detection on a mobile device with modest hardware specifications?

\subsection{Overview of Our Work}

We conjecture that the behavioural and contextual information pertaining to the use of a smartphone can be characterised by a diurnal cycle. ${ }^{3}$

As an example, mobile device user $A$ may be a university student who spends the evening at home and is on campus on most days. When user $A$ is at home or on campus, there will be familiar Bluetooth or Wi-Fi devices that the mobile device can sense. As user $A$ commutes to campus from home in the morning and back in the late afternoon, the mobile device can note the (changes in the) location of its owner. Furthermore, user $A$ may prefer to call or exchange text messages with certain contacts at certain times of the day - for example, during the lunch break on campus, or during the evening when at home. Finally, user $A$ may use certain applications

\footnotetext{
${ }^{3}$ For the record, weekly, monthly, and yearly cycles may exist as well, but are not investigated in this document.
} 
during particular times of the day, depending on the context.

We conjecture that the example in the previous paragraph can be generalised to other lifestyles as well. As a result of this conjecture, we introduce the notion of a time slice, which we obtain by dividing one day into equally sized $l$-hour-long slices. We conjecture that what the mobile device can sense during the $k$ th time slice of one day can be used to predict, with room for some error, what happens on another day's $k$ th time slice.

The time slice model can be used to detect anomalies in the use of a mobile device. If time slice $k$ of "today" does not match the instances of time slice $k$ from a certain number of past days, it can be the case that the mobile device is being used by someone other than the owner, or it may be the case that the user is doing something unusual. In either case, a comfort-enabled device can take action to protect its owner and his/her data.

\subsection{Contributions}

The contributions of this thesis are:

- The time slice model, used for the summarization of the behavioural and contextual data sensed by a mobile device into data instances that can be compared with the data instances recorded in the past for anomaly detection purposes.

- Evaluation of the time slice model in conjunction with two of the three anomaly detection algorithms introduced by Eskin et al. in [3], where we compare the accuracy of the time slice model across two data sets, namely the Reality Mining and Social Evolution data sets, and against models that do not consider temporal information.

- A comparison of the contribution of the features available in the Reality Mining 
and Social Evolution data sets to the accuracy of the time slice model used in conjunction with the aforementioned anomaly detection algorithms.

- The deployment of the time slice model and the aforementioned anomaly detection algorithms on an actual mobile device, showing their feasibility in resourceconstrained settings, via a qualitative user experience experiment carried out by the author, and the quantification of the resource overhead of the deployed approach.

\subsection{Thesis Structure}

This thesis document is organised as follows:

We begin by introducing concepts related to our work in the Background chapter, such as a discussion of the three types of anomaly detection approaches, two anomaly detection algorithms on which we base our methodology, performance metrics used in the evaluation of our methodology, and the types of fusion in multimodal biometrics.

Next, in the Related Work chapter, we review work that is closely related to our application domain and methodology, and end the chapter with a comparison of the related work in tabular form.

Afterwards, in the Methodology chapter, we first introduce the two data sets we use for the evaluation of our methodology, the features that the data sets contain, and which features we choose for anomaly detection. Following is a discussion of the data model and distance function we use for anomaly detection purposes. Finally, we end the chapter with a motivation and an explanation of our match score level fusion method.

In the Evaluation chapter, we report the results of the evaluation of our methodology using the two data sets which were discussed in the Methodology chapter, and 
discuss how various parameters and variables affect the evaluation results. The chapter ends with a comparison of our methodology to one of the most related works in terms of the data set, features and evaluation metrics which were used.

In the Deployment chapter, we briefly cover our experience in the evaluation of our work deployed on an actual mobile device.

Finally, the thesis ends with a Conclusion chapter, outlining our findings and future work. 


\section{Chapter 2}

\section{Background}

In this chapter, we review concepts we make use of in our work. We begin by introducing types of anomaly detection approaches, and note the category in which our work falls. Afterwards, we briefly cover the algorithms that we use for anomaly detection purposes, followed by the performance metrics used for evaluation. Finally, we provide a short discussion of fusion methods used in biometric systems.

\subsection{Types of Anomaly Detection Approaches}

In [4], Chandola et al. note the existence of three types of approaches that can be used to perform anomaly detection:

- Supervised Anomaly Detection, in which a training data set containing labelled normal and anomalous data is utilized to build a machine learning model in order to recognise anomalies in the test data set.

According to [4], the two major disadvantages of supervised methods are (1) the need to obtain accurately labelled data describing anomalies, and (2) the existence of class imbalance in the training data set, where the number of anomalous data items may be much smaller compared to the number of normal data items, due to the nature of the problem. The first disadvantage is especially important 
in our application domain, where labelled anomalous behavioural pattern data may be very difficult to obtain.

Another disadvantage that we find important is the fact that with a supervised approach, there exists a need to deploy with the anomaly detection software anomalous behavioural patterns on a mobile device in addition to its legitimate user's behavioural patterns.

- Semi-Supervised Anomaly Detection, where the training data is expected to contain only normal data, or in our case, data belonging to the legitimate user or owner of the mobile device.

- Unsupervised Anomaly Detection, where the approach operates under the assumption that the anomalous data items are a lot less frequent (or prevalent) when compared to the normal data items. As noted in [4], unsupervised anomaly detection approaches do not require training data.

Our methodology falls under the "semi-supervised anomaly detection" category, where, as the training data set, we provide the anomaly detection algorithm with the data produced by the interactions of a legitimate user with his/her mobile device only.

\subsection{Two Anomaly Detection Algorithms}

As we have noted, our work utilizes a semi-supervised approach. The algorithms we make use of are the first two algorithms introduced by Eskin et al. in [3], where the authors discuss the use of three algorithms for unsupervised anomaly detection: (1) Fixed-width clustering, (2) an optimized variant of k-Nearest Neighbours, and (3) the one-class Support Vector Machine (SVM).

These algorithms are based on the assumption that (1) anomalous data instances are rarer than normal data instances, and that (2) anomalous data instances are 
"qualitatively different" than normal data instances.

\subsubsection{Fixed-Width Clustering Algorithm}

The first algorithm approximates the number of data instances within distance $w$ of a data instance $x$ in the feature space, which can be used to estimate whether $x$ is in a sparse region of the feature space.

Ideally, we would like to know the density of a ball with a radius $w$ around a certain data instance in the feature space in terms of the number of instances in the ball, which is expressed with $N(x)$. However, calculating $N(x)$ for all instances in a set has $O\left(N^{2}\right)$ complexity because of the need for pairwise instance distance calculations. [3]

To avoid a complexity of $O\left(N^{2}\right)$, Eskin et al. propose to approximate $N(x)$ via the use of fixed-width clustering. Each instance in the feature space can be clustered around a cluster center (as detailed below) and the number of instances around a cluster center $c$ could be called $N(c)$. It is possible to approximate $N(x)$ with $N(c)$, where $x$ is a data instance in a cluster whose center is the instance $c$. While this approximation may be inaccurate with highly overlapping clusters, for sparse areas, it will be more accurate.

In this clustering algorithm, every data instance is compared to the centers of the currently available clusters using a distance function. The data instance in question is added to all clusters whose centers have a distance $w$ or less to the data instance. If no clusters are suitable according to this criterion, a new cluster is created with the current data instance as the new cluster's center. ${ }^{1}$ Finally, at the end of the clustering process, all data instances belonging to "small" clusters are considered anomalous, where the definition of "small" is based on the number of data instances in a cluster,

\footnotetext{
${ }^{1}$ This fixed-width clustering algorithm is dependent on the order of the data instances which are clustered.
} 
and is a threshold value set by the operator.

As explained above, the algorithm expects two parameters: the cluster width $w$ and a threshold anomalous cluster size.

The computational complexity of the fixed-width clustering algorithm is not provided by Eskin et al. in [3]; however, intuitively we can state that the computational complexity is $O\left(N_{c}\right)$, where $N_{c}$ denotes the number of clusters obtained after the clustering process. Because the number of clusters is dependent on the cluster width parameter, the computational complexity will decrease as the cluster width is increased, and the complexity will increase as the cluster width is decreased. Nevertheless, because the number of clusters will always be less than the number of data instances with reasonable cluster width parameter values, we can note that $O\left(N_{c}\right)$ will be smaller than $O(N)$.

\subsubsection{An Optimized k-Nearest Neighbours Algorithm}

Eskin et al.'s k-Nearest Neighbors (k-NN)-based anomaly detection algorithm determines whether a data instance in the feature space is in a sparse region by adding the distances of the instance to its $k$ nearest neighbors, which is called the $\mathrm{k}-\mathrm{NN}$ score. If this score is larger than a threshold value, the instance to whom the score belongs is considered anomalous. As $\mathrm{k}-\mathrm{NN}$ is based on pairwise inter-instance distance calculations, it has $O\left(N^{2}\right)$ complexity in unsupervised settings.

Eskin et al. propose the use of "canopy clustering," where the feature space is divided into sub-spaces "so as to remove the necessity of checking every data [instance]," which avoids the need for $N^{2}$ distance calculations. [3]

The k-NN algorithm starts with a slightly modified version of the fixed-width clustering algorithm discussed in Section 2.2.1, where the modification involves assigning each data instance to at most one cluster. After the clustering is complete, the authors make use of the following inequalities, where $w$ is the radius of a cluster 
"ball":

If $x_{1}$ and $x_{2}$ are in the same cluster, then we can write the following to note that the distance between the two data instances is less than or equal to the diameter of the "ball":

$$
d\left(x_{1}, x_{2}\right) \leq 2 * w
$$

If $x_{1}$ and $x_{2}$ are in different clusters, then:

$$
\begin{aligned}
& d\left(x_{1}, x_{2}\right) \geq d\left(x_{1}, c\left(x_{2}\right)\right)-w \\
& d\left(x_{1}, x_{2}\right) \leq d\left(x_{1}, c\left(x_{2}\right)\right)+w
\end{aligned}
$$

In the inequalities above, $c\left(x_{i}\right)$ refers to the center of the cluster which contains the data instance $x_{i}$.

Let us consider three sets, $C$ (the set of all clusters), $P$ (the set of potential nearest neighbors) and $K$ (the set of $k$-nearest neighbors). At first $P$ and $K$ are empty.

For a data instance $x$, a cluster is chosen from $C$ such that the chosen cluster's center is the closest to $x$. Then the chosen cluster is removed from $C$ and "opened," where all of the data instances in the chosen cluster are added to $P$.

Afterwards, the distance of each data instance in $P$ to $x$ is compared to a threshold value, $d_{m i n}$, which is defined as:

$$
d_{\min }=\min _{c \in C} d(x, c)-w
$$

where $d(x, c)$ is the distance of the data instance $x$ to the center of the cluster $c$. If the result of the comparison is such that the distance between $x$ and a data instance $x_{i}$ in $P$ is less than $d_{\text {min }}$ (i.e., $\left.d\left(x, x_{i}\right)<d_{\text {min }}\right)$ then the instance $x_{i}$ is moved from set $P$ to set $K$. If this condition is not satisfied by any data instance in $P$, then the next closest cluster is "opened" up and all of its data instances moved to $P$. We 
should note that the value of $d_{\min }$ will increase because of this "open-up" operation. The algorithm terminates when there are $k$ data instances in the set $K$.

Eskin et al. note that in this algorithm most of the processing time is spent on calculating distances between cluster centers and $x_{i}$, and that the choice of $w$ affects the efficiency of k-NN score computations but not the k-NN scores themselves.

Eskin et al. do not provide a computational complexity analysis of this algorithm in [3]; however, we can intuitively and empirically (based on our experience) state that the complexity of this method is smaller than $O\left(N^{2}\right)$.

\subsubsection{Discussion}

We choose to use the two algorithms discussed above because they are distance-based anomaly detection algorithms that can make use of custom distance functions to calculate the distances between pairs of data instances. As the reader will see in the Methodology chapter, the data instances we classify are complex data structures which need unstraightforward distance functions.

\subsection{Performance Metrics in Machine Learning}

In classification problems, a machine learning algorithm makes a prediction on the label of a data point, which may or may not be the same as the actual label of the data point in question. Based on the agreement (or disagreement) between the prediction of the classifier and the actual label, there are four different possibilities. We present these four possibilities in Table 2.1, which is called the confusion matrix.

We follow the convention that the data items which we are looking to find are labeled as positive, and the others as negative. For example, in an intrusion detection setting, if a data point is associated with an attack, it would be labeled positive, and a data point not associated with an attack would labeled negative. 


\begin{tabular}{|c|c|c|c|}
\cline { 3 - 4 } \multicolumn{2}{c|}{} & \multicolumn{2}{|c|}{ Label } \\
\cline { 3 - 4 } Prediction & Positive (Attack) & Negative (Not an Attack) \\
\cline { 2 - 4 } & Negative (Not an Attack) & False Negative & False Positive \\
\hline
\end{tabular}

Table 2.1: Confusion Matrix

If a classifier - for example, an Intrusion Detection System (IDS) - predicts that a data point represents an attack, and the data point's actual label indicates an attack, then the classifier is said to achieve a True Positive, which is the upper left cell in the confusion matrix. Conversely, if the IDS predicts that a data point is associated with an attack, but the data point's actual label indicates otherwise, then the IDS achieves a False Positive, as noted by the upper right cell of the confusion matrix.

Based on the confusion matrix, one can devise a number of performance metrics, one of which is Accuracy. Accuracy is calculated by adding the number of true positives and true negatives, and dividing this quantity by the sum of the number of true positives, false positives, false negatives and true negatives. In more compact form:

$$
\text { Accuracy }=\frac{|T P|+|T N|}{|T P|+|F P|+|F N|+|T N|}
$$

We should note that accuracy is not suitable when a class imbalance exists in the data, where, for example, the data set has a large amount of "normal" (negative) data points and a very small amount of data points associated with "attacks" (positives). In such a scenario, consider an IDS which always predicts "normal" with a data set whose $99 \%$ is composed of negative data points, and $1 \%$ is composed of positive data points. In this case, the accuracy of the IDS would be 99\%, even though the IDS is not able to recognise any attacks.

Other metrics include Recall (also known as True Positive Rate, or TPR), False 
Negative Rate (FNR), and False Positive Rate (FPR). These metrics do not suffer from the class imbalance problem because they only make use of a single column of the confusion matrix, which guarantees that the ratios represented by the metrics stay the same even in the case of class imbalance. [5, p. 10]

Continuing with our IDS example, Recall, or True Positive Rate, measures the ratio of the actual attacks that were predicted as attacks by the IDS over the total number of actual attacks.

$$
\text { Recall }=\mathrm{TPR}=\frac{|T P|}{|T P|+|F N|}
$$

The False Positive Rate is the ratio of the number of incorrectly predicted attacks to the number of all data points not associated with an attack.

$$
\mathrm{FPR}=\frac{|F P|}{|F P|+|T N|}
$$

Finally, False Negative Rate is the ratio of the number of incorrectly predicted non-attacks over the total number of actual attacks.

$$
\mathrm{FNR}=\frac{|F N|}{|T P|+|F N|}
$$

The three metrics we have just mentioned can be plotted in two configurations to obtain a visual presentation of the trade-offs associated with the tuning of a classifier, which might, for example, have a "knob" that controls the trade-off between false positives and false negatives.

In the first configuration, FPR and FNR can be plotted against a "threshold," which represents the "knob" of the classifier. An example of such a plot can be seen in Figure 2.12.

\footnotetext{
${ }^{2}$ Adapted from [6] and [7, p. 32].
} 


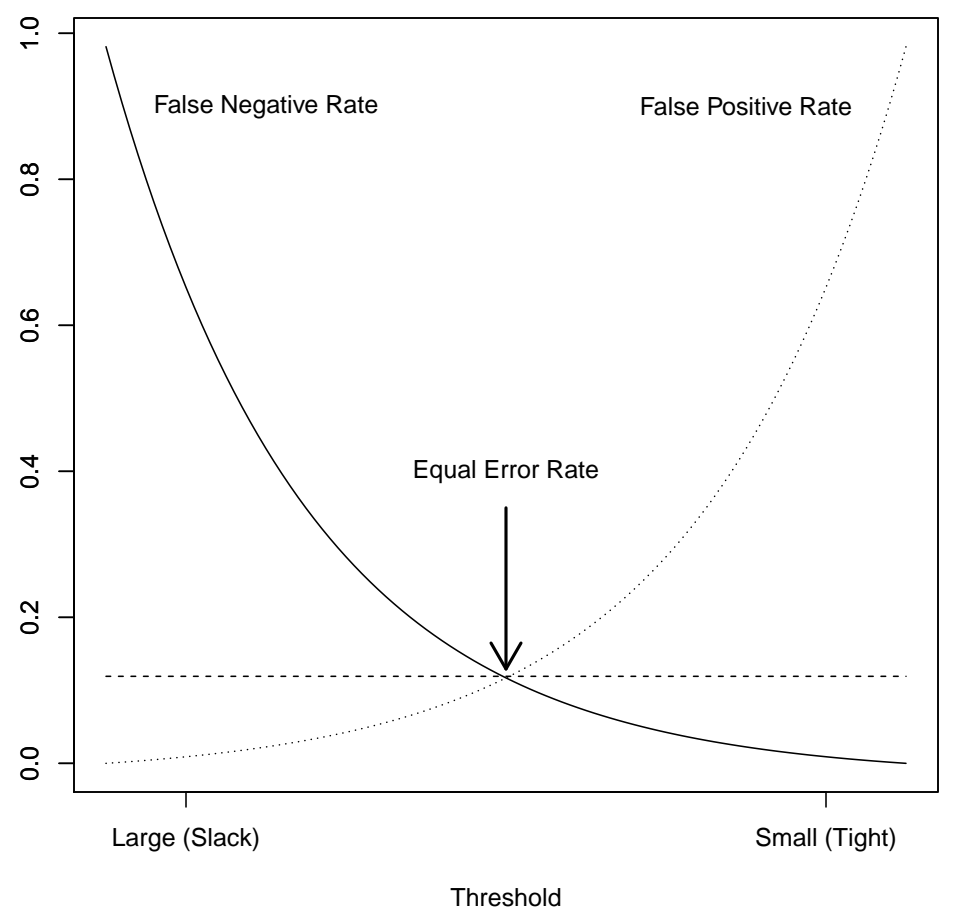

Figure 2.1: A Fictitious Plot of FPR and FNR against Threshold ${ }^{2}$

As can be seen in Figure 2.1, the Equal Error Rate (EER) is the error rate at which FPR equals FNR. As can be guessed from its name, the lower the EER value of a classifier, the higher its performance. EER is used as a summary performance metric in some of the related work we review.

In the second configuration, TPR (or Recall) can be plotted against FPR to obtain a curve called the Receiver Operating Characteristic (ROC) curve. The area under the ROC curve is called the $A U C$, a higher value of which indicates better performance. AUC is another summary metric used in related work.

A sample ROC curve can be seen in Figure 2.2. In the figure, the diagonal line with positive slope indicates a classifier whose performance is equal to that of random guessing. The AUC value associated with such a classifier is 0.5 . The FPR at the point of intersection of the ROC plot with the diagonal line with negative slope 


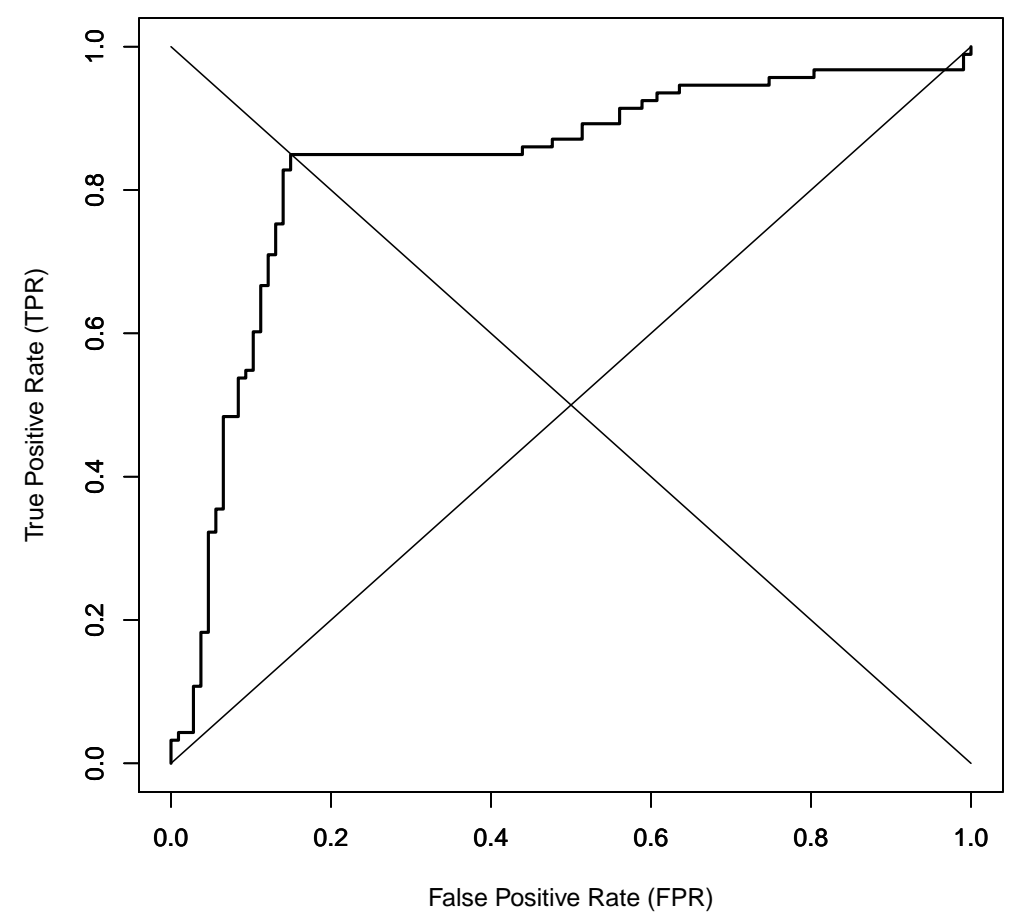

Figure 2.2: A Receiver Operating Characteristic (ROC) Curve

is the EER.

Finally, we should note that AUC is not affected by the class imbalance problem. [5] Furthermore, because EER is a summary metric obtained from the ROC curve, and because according to [5] ROC curves are not affected by class imbalance, we assume that EER is not affected by class imbalance either.

Because the evaluation of our methodology involves class imbalance, we shall use the AUC and EER performance metrics in our work.

\subsection{Fusion Methods in Biometrics}

As noted before, we use the behavioural patterns of a mobile phone user in a biometric verification setting. Given that we make use of different aspects of a mobile phone 
user's behaviour, such as phone call patterns, spatio-temporal patterns, and so on, the selection of a method for combining - or fusing - these different behavioural patterns becomes relevant.

According to Ross and Jain [8], there are three methods for biometric fusion.

- Feature Level Fusion, where the feature vectors generated by each biometric sensor are concatenated to produce higher-dimensional feature vectors, which, in our case, can be presented to a machine learning classifier.

- Match Score Level Fusion, where the feature vectors for each biometric sensor are classified, and the scores resulting from the classification of the feature vectors are combined. A score, in our case, may be the likelihood that the current user of a mobile phone is its owner, based on the features generated by a sensor.

- Decision Level Fusion, where the decisions resulting from the classification of individual feature vectors are combined. One way of combining decisions is voting.

In our work, we use feature level and match score level fusion methods. In the former, we consider the features from multiple sensors as one data instance, whereas for the latter we consider each feature as a data instance on its own.

\subsection{Conclusion}

As we have noted in this chapter, according to the criteria discussed in [4], our work falls within the group of semi-supervised anomaly detection methods.

As will be seen in the next chapter, various original and traditional anomaly detection algorithms are used in related work. We choose to use two algorithms introduced by Eskin et al. in [3]. 
Furthermore, because of the existence of class imbalance in our evaluation scheme, we use AUC and EER as the performance metrics, which are used in related work as well.

Finally, to combine information from multiple sensors, we make use of feature level and match score level fusion methods. 


\section{Chapter 3}

\section{Related Work}

In this chapter, we review work that is related to anomaly detection and Device Comfort. In the related work that we review, authors make use of a number of different features, machine learning algorithms, evaluation strategies and performance metrics to achieve a similar goal: the use of anomaly detection for the security of a personal computing device, which is a mobile phone for most proposals, but in some cases is a laptop or a desktop computer.

In Section 3.1, we provide a brief introduction to the related work. In the following sections of the chapter, we review the related work in terms of the different aspects of the proposals:

- What is the targeted personal computing platform? For example, is the proposal concerned with the security of a mobile phone or a desktop?

- Has the proposal been implemented and deployed?

- What are the features used by the proposed anomaly detection method?

- Are multiple types of features "fused" together to obtain a multimodal approach?

- What is/are the machine learning algorithm(s) used to implement anomaly detection? 
- Is the machine learning algorithm a one-class or a two-class learning algorithm?

- Which data set is used for evaluation? Is the evaluation made with a data set available to the research community, or with an original data set collected by the authors?

- How is the proposal evaluated?

- Does the evaluation method consider changing habits of the personal computing device user?

- Which performance metrics are used for evaluation purposes?

\subsection{Introduction}

The proposals we review are made by the following authors, in alphabetical order: Branscomb [9], Crawford [7], Li [10,11], Shi et al. [12], Yazji et al. [13,14], Yazji et al. [15], and finally Zhu et al. [16]. These proposals were found in related journals such as Personal and Ubiquitous Computing (PUC) and the proceedings of related conferences such as Ubiquitous Intelligence and Computing (UIC). The references sections of some of the related work items have been another important resource.

Below, we briefly introduce the above-mentioned authors' proposals.

To verify whether the user of a mobile phone is its owner or not, Branscomb proposes to use the number of seconds that the user spends in each application category for the duration of a session, which starts with the switching on of the mobile phone's screen and ends with the switching off of the mobile phone's screen.

In Crawford's work, a mobile phone user is verified to be the owner of the mobile phone or not based on keystroke dynamics and voice verification techniques. Crawford proposes a "transparent authentication framework," which has similarities to Marsh 
et al.'s Device Comfort concept.

Li's "behavioural profiling" approach uses different features, where location of the mobile phone, and application usage, phone call and text messaging patterns in the mobile phone user's behaviour are utilized as behavioural biometrics to verify the identity of the mobile phone user.

In contrast, Salem et al. are concerned with the security of a personal (desktop) computer for masquerade detection, ${ }^{1}$ where the search-related behaviour of a computer user in terms of the file-system accesses are considered.

Shi et al. name their approach "implicit authentication," and target mobile phones, where they model the owner's behaviour via his/her usage of the mobile phone in terms of time, location, web browsing history, and phone call and text message histories.

We review two proposals by Yazji et al., the first of which considers the spatiotemporal patterns in a mobile phone user's behaviour only. The second - and earlier - proposal investigates whether the patterns in network accesses and the use of a file-system on a laptop computer are sufficient to verify the identity of the user.

Finally, Zhu et al.'s proposal takes a different approach on behavioural biometrics, where the motions (sensed by accelerometers and other sensors) that a mobile phone user makes are verified to secure the mobile phone in question.

\subsection{Targeted Platforms and Implementation Sta- tus}

Yazji et al. make two proposals, one in $[13,14]$ and another one in [15]. The first two publications are concerned with the security of a mobile phone, whereas the third

\footnotetext{
${ }^{1}$ Masquerade detection is the detection of attackers who assume the identity of an authorized user, who might have, for example, left his/her workstation unattended.
} 
one considers the security of a laptop computer, even though it is also applicable to desktop computers. In all of the proposals made by Yazji, a server works hand-inhand with the mobile device in question to achieve the security of the mobile device, where computation and energy intensive tasks of the anomaly detection schemes are off-loaded to the server.

This is in contrast to the other work we have reviewed, all of which propose to keep the processing on the mobile device itself. Crawford notes in $[7$, p. 37] that sending data to a remote server to perform anomaly detection may have privacy implications.

Branscomb, Crawford, Li, Shi et al., and Zhu et al. all target mobile phones. The proposal by Salem et al. targets desktop computers.

Implementation and Deployability Status To the best of our knowledge, of the reviewed work, only Branscomb and Zhu et al. have implemented fully deployable solutions, which contain both the data collection and the anomaly detection logic, and which were both for Google's Android. ${ }^{2}$

In comparison, Yazji et al., with their mobile phone-related work in [13,14], and Li have only implemented the anomaly detection procedures without deployable code.

Furthermore, we see that Crawford, Salem et al., Shi et al. and Yazji et al. [15], have deployed only the data collection related parts of their proposals and performed the data analysis offline. Of these authors, Crawford has implemented two applications for Apple's iOS, Shi et al. for Google's Android, and Salem et al. and Yazji et al. for Microsoft's Windows operating system.

\footnotetext{
${ }^{2}$ We should note that Branscomb's implementation with the anomaly detection logic is a proofof-concept version as described in [9, p. 20], whereas Zhu et al.'s implementation was deployed for a three-day-long user study.
} 


\section{$3.3 \quad$ Features}

Anomaly detection on personal computing devices can be performed using different features, sometimes in a multimodal fashion.

Branscomb groups applications available on mobile devices running Google's Android into different categories, and looks at the number of seconds that the user spends in each application category during a "session," which is defined as the time between the switching on of the mobile phone screen and until the switching off of the screen. The used features include the number of applications launched per category, and the duration (number of seconds) for which each application category has been used. Branscomb also uses some temporal information in the form of a feature that indicates whether the usage occurs on a weekday or not, with the assumption that mobile phone users may use different applications during weekends.

Crawford's approach makes use of keystroke dynamics and voice patterns which are used to detect whether a mobile device is being used by its owner, where the inter-key delay and the key hold time are used for keystroke dynamics verification. For voice verification, a number of aural/audial features generated by a commercial software package are used.

In their proposal, Zhu et al. use accelerometer, gyroscope and magnetometer readings of a mobile phone, where the readings are split into two-second-long time quanta, each of which is described in terms of the following statistical features describing the readings: Root Mean Square, Root Mean Square Error, minimum and maximum values, average change between samples, the number of local peaks and crests, and others.

Yazji et al., in $[13,14]$, use as features only the location of a mobile phone in terms of cell tower area and cell identifiers, and the time of day to find anomalies in the spatio-temporal patterns of the user of a mobile phone. 
Shi et al. and Li make use of other features in addition to time of day and location. Shi et al. make use of time of day, the web browsing history, phone call and text message exchange logs, and the location of a mobile phone (via the mobile phone's Global Positioning System sensor).

Li et al. make use of what they call inter-application features and intra-application features, where the inter- features refer to the different applications used by the user, and the intra- features refer to the features generated by the user's calling a phone number inside a telephony application, or the user's sending/receiving a text message inside a text messaging application. Another feature that is used by Li et al. is the location of the mobile device (as acquired from cellular towers in the form of area and cell identifiers).

Finally, Yazji et al., in [15], and Salem et al. take a relatively different approach by considering the accesses made by a user using a laptop or a desktop, respectively. Yazji et al. consider file-system and network accesses made by the user, and the name of the process used to make the access. These features are collected for the duration of the training period, and every five minutes during the deployment/testing phase.

Salem et al. are inspired by the fact that a user and a masquerading attacker have different "search" related behaviours when accessing files on a file-system, where an attacker masquerading as a legitimate user would need to explore the file-system with appropriate programs in order to find files with valuable information. Accordingly, Salem et al. categorize Windows applications into more than twenty categories, ${ }^{3}$ and they take into account every two-minute-long quanta of user behaviour, the activity in each of which is described using the following features: (1) the number of search-related file-system access records, (2) the number of file touches, and (3) the percentage of user actions involving file-system navigation.

\footnotetext{
${ }^{3}$ Some of the application categories are: searching, file-system management, development, networking, configuration, and browsing.
} 
Multimodal Approaches Of the proposals mentioned above, most are multimodal, in the sense that they make use of different kinds of behavioural biometrics belonging to the owner of a personal computing device in order to verify whether the device is being used by its owner or not.

We notice that Yazji et al. (in $[13,14]$ ), Branscomb and Salem et al. use a unimodal behavioural biometric, by considering only spatio-temporal patterns, application usage patterns, and file-system access patterns, respectively.

In comparison, Crawford, Li, Shi et al., and Yazji et al. (in [15]) make use of multimodal behavioural biometrics, where they fuse together features coming from different "sensors." For example, Crawford fuses keystroke dynamics and voice patterns using match score-level fusion, whereas Li uses feature-level fusion and via the use of a "smoothing function," decision-level fusion. ${ }^{4}$ Based on our understanding, Shi et al. and Yazji et al. also use match score-level fusion.

Finally, we believe Zhu et al. [16] use feature-level fusion, where they concatenate the feature vectors obtained from the accelerometers, gyroscopes and magnetometers into a single higher-dimensional feature vector.

\subsection{Methodologies}

In this section we cover the (machine learning) methods used by the authors of the related work. Some authors choose to use standard machine learning algorithms, whereas others come up with their own empirical anomaly detection schemes. Even though the majority of the cases involve one-class learning approaches, we also found proposals making use of two-class learning.

As Wang and Stolfo note in [17], one-class learning can be just as powerful as

\footnotetext{
${ }^{4}$ Aside from noting in $[10$, p. 310$]$ that the smoothing function is similar to a "winner-takes-all decision-based biometric fusion model," Li does not explain in detail how the smoothing function works.
} 
two-class learning in anomaly detection applications, and in fact, one-class learning may be more suitable for such applications, based on the fact that one-class machine learning algorithms need only the data belonging to the "normal" user. We should also note that it can be difficult (if not impossible) to obtain the behavioural patterns of attackers.

\subsubsection{Branscomb}

Branscomb chooses to use the IBk (k-Nearest Neighbours) and Naive Bayes two-class classifiers that are available in the Weka [18] machine learning framework. During training and testing, Branscomb provides the mentioned machine learning algorithms both the authorized user's and the attackers' data.

\subsubsection{Crawford}

Similar to Marsh et al.'s proposed Device Comfort level dynamics in [1], Crawford proposes a scheme where if the mobile device is not being used, its level of confidence gradually diminishes, whereas with correct explicit or implicit authentication, the confidence level rises. The confidence of the mobile device depends on behavioural biometrics. The following two-class machine learning algorithms are used to prove the possibility of using keystroke dynamics and voice verification as behavioural biometrics in Crawford's framework: Decision Tree, Naive Bayes (with Gaussian and Kernel Density Estimation distributions) and k-Nearest Neighbours (with Manhattan and Euclidean distance metrics). In particular, Neural Networks and Support Vector Machines are not considered because of their computation-intensive nature. 


\subsubsection{Li et al.}

Li et al.'s empirical anomaly detection algorithm makes use of the ratio of the number of times that a feature value was observed during the training period over the sum of the number of times that all feature values were observed during the training period. The algorithm also assigns weights to individual features based on their predictive power. Formally, the main equation of the algorithm can be seen in Equation 3.1.

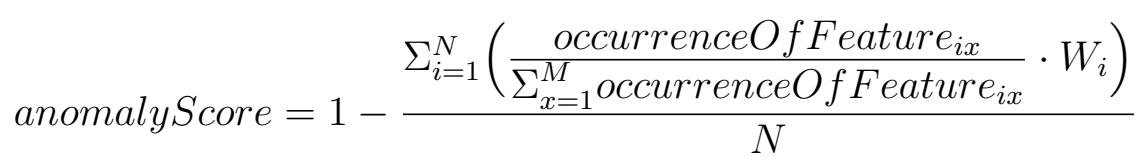

where $i$ represents the features of the application the data from which is being classified, $N$ is the number of features, $x$ is the value of feature $i, M$ is the number of values for feature $i$, and $W_{i}$ is the weight of feature $i$.

For example, if the telephony application is being used, then the following features are recorded: phone number and the location at which the phone call is made. In this example, $N$ is 2 and the value of feature $_{1}$, is the phone number that is called, and feature $_{2}$ is the location. ${ }^{5}$ Interestingly, Li et al. ignore the time of day, which is described in [11] as negatively contributing to the overall performance of their method.

Although not shown in Equation 3.1, Li et al.'s algorithm uses a smoothing function, where a configurable number of anomaly decisions for consecutive events are grouped together to collectively determine whether the mobile device is being used in an anomalous manner. ${ }^{6}$

Finally, we should note that Li et al. use their algorithm in one-class mode, where they have only the normal user/owner's data available during the training phase.

\footnotetext{
${ }^{5}$ As the reader will notice, $\mathrm{Li}$ et al. use feature-level fusion here by fusing the location and the phone call features.

${ }^{6}$ Unfortunately, we were not able to find in [10] or [11] details about how the smoothing function works.
} 


\subsubsection{Salem et al.}

Salem et al. utilize the one-class Support Vector Machines classifier.

\subsubsection{Shi et al.}

Shi et al. model a user's behaviour as follows: for web browsing history, phone call contacts and text messaging contacts, Shi et al. introduce "good" and "bad" contexts. "Good" contexts are, for example, known (that is, in-contacts-list) phone numbers contacted during the training period, whereas "bad" phone call contexts are phone calls to unknown phone numbers. A classifier is trained on the probability of time elapsed since the last "good" context based on the time of day, and the number of "bad" contexts in the past "N" hours based on the time of day. For GPS location data, the Gaussian Mixture Model clustering algorithm based on Expectation Maximization is used to cluster each 6-hour epoch of each day of the mobile phone's location information.

For anomaly detection, Shi et al. assume that the probability of each feature taking on a value based on the current time of day is independent. Based on the probability distribution obtained from the training data, Shi et al. calculate the probability of individual features taking on their values at the current time of day, multiply the probabilities and compute a "score," which is compared to a threshold value to decide whether the user's behaviour is anomalous or not.

Because the scoring algorithm is dependent on the time of day, the score decays over time if the mobile phone is not used, if no "good" events occur, or if "bad" events occur. This is similar to the dynamics of the Device Comfort level as explained by Marsh et al. in [1].

Since Shi et al. use only the authorized user's data during the training phase, they effectively use a one-class classifier. 


\subsubsection{Yazji et al.'s Spatio-Temporal Anomaly Detection Pro- posal}

One method used by Yazji et al. is an empirical and collective ${ }^{7}$ anomaly detection scheme, where the authors build a matrix in which each column corresponds to a major location in the user's spatio-temporal patterns, and each row corresponds to the time of the day with a precision of one minute. Each cell in the matrix contains the probability of the user being at a certain location (indicated by the column) at a certain time of day (indicated by the row). Anomaly detection is performed by considering 5-minute long traces of a user's behaviour, where the values of the relevant cells of the matrix are added and the resulting sum is compared to a threshold value.

Yazji et al. also use a second anomaly detection method, which is similar to a Hidden Markov Model. More information about the second method can be obtained from [13] and [14].

This proposal is also one-class in nature, as the "training matrix" is composed of only the data of the owner of the mobile phone.

\subsubsection{Yazji et al.'s File-System and Network Access-based Anomaly Detection Proposal}

In this proposal, Yazji et al. first map the categorical features (file-system hierarchy and IP addresses) to numerical features, and afterwards they use the K-Means clustering algorithm with 8 cluster-centres and the Euclidean distance function to cluster the laptop owner's file-system and network access patterns separately.

After a maximum of 20 iterations, the K-Means algorithm clusters the input data, and the percentage of the number of data points in each cluster is obtained to form

\footnotetext{
${ }^{7}$ According to [4], a collective anomaly detection scheme is one where groups of data items are found anomalous, as opposed to the identification of individual outlier data items.
} 
a "Distribution Vector."

Anomaly detection is carried out by comparing the distribution vector obtained from the training data to the distribution vector obtained from the testing data using

the Euclidean distance. If the Euclidean distance function produces an anomaly score above the configurable threshold, then the testing data is considered to have been produced by the file-system and network accesses made by an attacker.

Yazji et al.'s file-system and network access-based anomaly detection proposal is also one-class in nature, because the training period is only concerned with the authorized laptop user's data.

\subsubsection{Zhu et al.}

Zhu et al. use an $n$th order Markov model - also called an $n$-gram model - to process a mobile phone user's motions similar to the way language is processed with such a model.

The authors note that language and motions share some similarities, where a user's atomic movement (such as tilting a device) might be considered a letter, a movement (such as picking up a phone) considered a phrase, and an action (placing a phone call) considered a sentence.

Based on this similarity, the authors use an $n$-gram model to predict which "word" comes after a "phrase" in terms of the user's motions. This is achieved by converting raw sensor readings for each two-second-long time quanta to feature vectors.

After the training period is complete, the feature vectors are clustered into $V$ (which was chosen to be 200) clusters, the centers of which are considered a "vocabulary" of behaviour labels.

During the testing phase, the likelihood that a behaviour $l_{i}$ comes after a particular sequence of $n$ behaviours is approximated using the following equation: 


$$
P\left(l_{i} \mid l_{i-n+1}^{i-1}\right)=\frac{C\left(l_{i-n+1}, \ldots, l_{i-1}, l_{i}\right)}{C\left(l_{i-n+1}, \ldots, l_{i-1}\right)}
$$

Based on our understanding, during the testing phase a sequence of feature vectors $l_{i}$ are obtained every two seconds. Afterwards, this equation counts the number of occurrences in the training data of the sequence represented in the numerator and divides the resulting count by the number of occurrences in the training data of the sequence represented in the denominator. ${ }^{8}$

Afterwards, a "sureness" score for a string of $N$ behaviours is computed as follows: ${ }^{9}$

$$
P\left(l_{1}, l_{2}, \ldots, l_{N}\right)=\Pi_{i=1}^{N} P\left(l_{i} \mid l_{i-n+1}^{i-1}\right)
$$

Finally, an alarm can be raised if the sureness score is below a threshold value.

Because the training data is composed of only the authorized user's data, which is also noted by Zhu et al. in [16], the method used by the authors is a one-class approach.

\subsection{Data Sets}

We see that out of the proposals we have reviewed, only three used a publicly available data set. In one of these three cases, the data set had been published but is no longer available.

Yazji et al.'s spatio-temporal anomaly detection proposal $[13,14]$ makes use of the temporal and spatial features of the Reality Mining ${ }^{10}$ [19] and the GeoLife [20] data

\footnotetext{
${ }^{8} \mathrm{Zhu}$ et al. do not describe in [16] what happens when a feature vector obtained in the testing phase does not perfectly match any vocabulary items. We assume that each testing phase feature vector is matched to the vocabulary item closest to the feature vector in question, using Euclidean distance, for example.

${ }^{9}$ The authors choose to use 5 as $N$ for evaluation, but they do not provide information about the chosen value of $n$, which is a different constant specifying how far into the "past" the $n$-gram should look in order to calculate a probability.

${ }^{10}$ We explain the features available in the Reality Mining data set later in this document.
} 
sets to evaluate their anomaly detection method. Li et al. also use the Reality Mining data set, but with the difference that in addition to the spatial feature, the phone call and text messaging features of the Reality Mining data set are utilized as well.

Salem et al. use the Are You You? (RUU) data set, collected by the same authors and made available in the past. ${ }^{11}$ The data set is a result of a user study with eighteen participants, where sensors installed on the participants' computers record timestamped file-system accesses, in addition to other features. To obtain data describing the behaviour of masquerading attackers, another user study with different participants is run, in which the participants are grouped according to type of intent: malicious, benign, and neutral. Each attacker participant is asked to behave according to their assigned intent.

The remaining proposals' authors all collect their own data sets, which have not been published.

Branscomb implements a prototype application for Google's Android to collect data. Because of technical difficulties, the data collected during the development of the prototype is used, which consists of the application usage patterns of six (6) participants.

Crawford implements for Apple's iOS one application to collect keystroke dynamics data, and another application to collect voice recordings, both for the purpose of data collection only. Two user studies are carried out to collect keystroke dynamics and voice-based biometric data from eight and nine participants, respectively, of which (based on our understanding) six participants are the same individuals in both studies. These six individuals' data is used for the multimodal behavioural biometrics feasibility study.

Shi et al. [12] choose to author and publish their own Android application and use the data made available by the users of the application. The data set is composed of

\footnotetext{
${ }^{11}$ We have not been able to find a copy of this data set online.
} 
data collected from 276 users, roughly 50 of which participated in the study for more than 12 days.

Yazji et al., for their file-system and network access-based anomaly detection proposal collect file-system and network access data from eight (8) participants over a period of two weeks.

Finally, Zhu et al. collected motion data from 20 volunteers over a duration of 72 hours, the first 24 hours of which is being used for training, the next 24 hours for testing with the same participants (to simulate day-to-day usage), and the last 24 hours for testing with different participants (to simulate attack scenarios).

\subsection{Evaluation}

In terms of evaluation, there are two schemes that arise depending on how the data set was collected: "1-versus-Rest" and "1-versus-Attackers."

The 1-versus-Rest method is carried out as follows: First, a data set is collected with $N$ participants. In the evaluation phase, one at a time, each participant is considered the "owner" of a personal computing device, and the rest of the participants are considered attackers.

We should note that the 1-versus-Rest suffers from some unrealism because the behaviour of the rest group may not always represent the behaviour of a real world attacker.

In contrast, the 1-versus-Attackers approach is carried out as follows: A data set is collected with $N_{u}$ participants who use their personal computing devices normally. Afterwards, another data set is collected with $N_{a}$ participants who attack a target personal computing device (which may or may not belong to one of the $N_{u}$ participants in the former data collection).

In the evaluation phase, one at a time, each participant belonging to the first $N_{u}$ 
individuals is considered the "owner" of a personal computing device, and the data from the $N_{a}$ attackers is used as positive (that is, attack) data.

\subsubsection{Salem et al.}

Of the reviewed work, Salem et al.'s evaluation method is, in our opinion, the most realistic one, precisely because they use the 1-versus-Attackers method in a rigorous manner.

Salem et al. were able to collect their own data set with "user" participants and other participants simulating three different kinds of attackers, who were given clear instructions about their modus operandi.

Salem et al.'s evaluation strategy is as follows: For each "user" participant, $80 \%$ of the data set is used for training purposes, and the remaining $20 \%$ of the data of the same user and the attacker participants' data is used for testing purposes.

We should note that Salem et al. use a static model, and as a result do not evaluate their proposal against concept drift, or in other words, drifting user habits.

\subsubsection{Yazji et al.'s File-System and Network Access-based Anomaly Detection Proposal}

Yazji et al. also use a 1-versus-Attackers method, except that their attacker scenarios are not as detailed as Salem et al.'s.

In [15], Yazji et al. explain that the attacker participants were the same participants as the user participants, with each participant "attacking" another participant's personal computing device. Furthermore, unlike Salem et al.'s "three different attacker profiles" approach, only one attacker profile was asked to be simulated from the participants: a modus operandi in which as much as possible valuable information should be targeted. 
Yazji et al. do not evaluate their proposal against drifting user habits either.

\subsubsection{Zhu et al.}

As was mentioned in the previous section, Zhu et al. provide a mobile phone trained with one participant's motions to another participant. ${ }^{12}$ As a result, their evaluation strategy is similar to Yazji et al.'s 1-versus-Attackers approach.

In addition, Zhu et al. do not evaluate their proposal against drifting user habits.

\subsubsection{Others}

While all of the remaining proposals make use of the 1-versus-Rest method, we should note that Shi et al. take an interesting evaluation approach, where they "splice" together an authorized user's data with different attackers' data as follows: For the location feature (which was collected in the form of GPS coordinates), they compute the delta between the user's and the attacker's locations, and then they add this delta to the attacker's location, so that from the machine learning algorithm's point of view, the attacker does not suddenly teleport with the mobile phone from one part of the world to another (which can be the case without this splicing approach, as Shi et al.'s data set contains data from participants living in different continents).

Of these proposals, only Li et al.'s proposal is evaluated such that concept/user habit drift is considered. To take into account drifts in the user's habits, Li et al. propose to build dynamic user profiles, where a user profile is generated from the part of the data set which comprises the 7,10 , or 14 days before the one-day-long part of the data set which is used for evaluation. This approach is equivalent to a scheme where a mobile phone "retrains" itself at every midnight so that the mobile phone takes into account only the past $D$ days of the user's behavioural patterns.

\footnotetext{
${ }^{12}$ That is, Zhu et al. permute the mobile phones among the participants.
} 


\subsection{Performance Metrics}

The reviewed work uses a variety of performance metrics ${ }^{13}$ to evaluate their proposals.

Li et al. report the performance of their proposal with EER. Crawford chooses to use AUC and EER, whereas Salem et al. use AUC only. Branscomb's proposal is evaluated with TPR, FPR, True Negative Rate (TNR), and FNR, while Yazji et al.'s file-system and network access-based anomaly detection proposal is evaluated with FPR and FNR. Zhu et al. make use of TPR and FPR metrics and ROC curves.

Yazji et al., with their spatio-temporal anomaly detection proposal, use $1-F N R$ as the performance metric, and call this "accuracy". 14

Finally, Shi et al. use an evaluation metric where they plot the number of times an attacker can use the mobile device without being detected against the number of times the authorized user can use the mobile device before being locked out.

\subsection{Conclusion}

We summarize our findings resulting from the literature survey in tabular form in Tables 3.1 and 3.2.

If we compare and contrast our work with the reviewed proposals, we can note the following:

We target mobile phones as most of the proposals do; however, because of the lack of time and resources to run a user study, we choose to use the Reality Mining and the Social Evolution data sets. Thus, the features we use for evaluation are limited to the ones in these data sets. ${ }^{15}$ Because we are using features from multiple sensors, our method is multimodal.

\footnotetext{
${ }^{13}$ Please see Section 2.3 for an overview of the metrics mentioned in this section.

${ }^{14}$ We quote accuracy here, because $1-F N R$ is not the definition of accuracy that is used in machine learning. Please see Section 2.3.

${ }^{15}$ These features are: application usage logs, Bluetooth and Wi-Fi discovery results, cellular location, and phone and text message exchange logs.
} 
In terms of similarities to our time slice model, we notice that some of the proposals also summarize the personal computing device user's behaviour into "time quanta." For example, Yazji et al., with their file-system and network access-based anomaly detection proposal, use five-minute-long time quanta. Similarly, Salem et al. use two-minute-long time quanta, whereas Zhu et al. use two-second-long time quanta. None of these researchers use the time of day as a feature.

Yazji et al., with their spatio-temporal anomaly detection proposal, consider the time of day in order to build a probability matrix indicating the times of the day at which the mobile device owner is present at one of his/her commonly visited locations.

Branscomb, and Li do not consider the time of day. Instead, Branscomb considers as a feature whether the use of the mobile device occurs on a weekday or during a weekend.

In terms of exploiting diurnal patterns, Shi et al.'s approach is similar, in part, to ours. Shi et al. build probabilistic models, based on the time of day, which indicate the number of hours since the last "good" event and the number of "bad" events in the past 24 hours. Shi et al. also use a clustering algorithm on the GPS coordinates of the user for each 6-hour-long epoch.

In terms of the remaining aspects, we deploy on an actual mobile phone the data collection logic and the anomaly detection logic, which can utilize either the fixedwidth clustering or the k-Nearest Neighbours algorithms in one-class mode.

For evaluation, because of the limitations of the data sets we are using, which do not have attacker data unlike some of the original data sets used by the reviewed work, we use the 1-versus-Rest method for evaluation.

Because of the existence of class imbalance in our evaluation method, we use the AUC and the EER metrics.

Finally, we evaluate our approach against drifting user habits as well. 


\begin{tabular}{|c|c|c|c|c|c|}
\hline 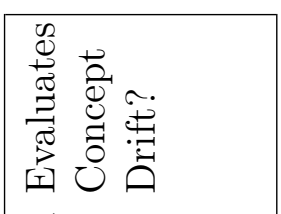 & $\stackrel{8}{z}$ & z & 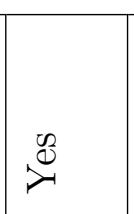 & z & z̊ \\
\hline & 密 & 竞 & 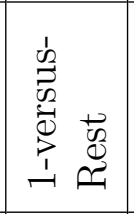 & 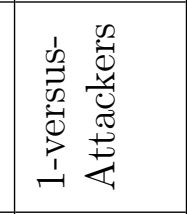 & 尊 \\
\hline 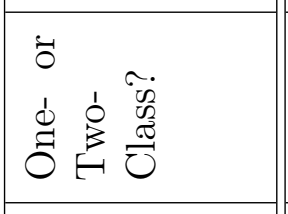 & 总 & 总 & $\Xi$ & 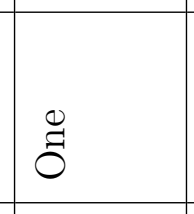 & 气 \\
\hline 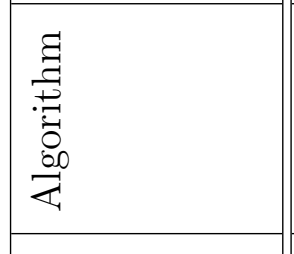 & 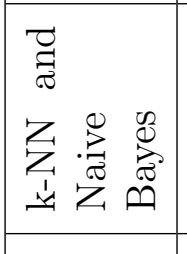 & 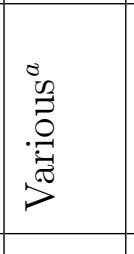 & 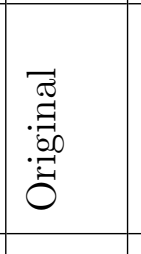 & 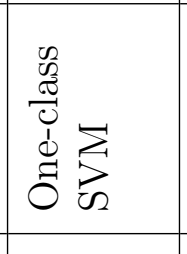 & 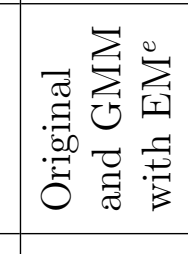 \\
\hline 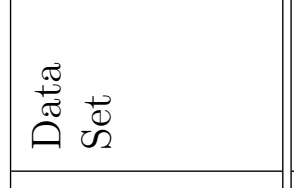 & 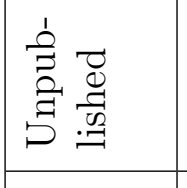 & 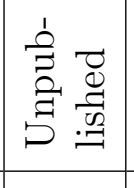 & 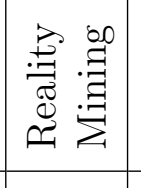 & 定 & 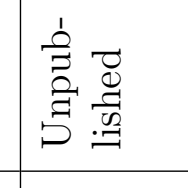 \\
\hline 吾 & ¿ & 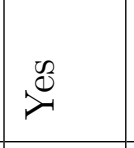 & $\underset{\sim}{\infty}$ & $\stackrel{8}{2}$ & $\stackrel{\infty}{\check{c}}$ \\
\hline 惹 & 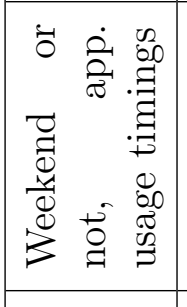 & 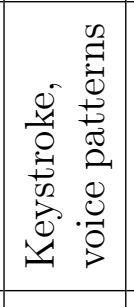 & 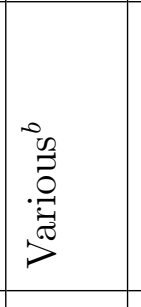 & 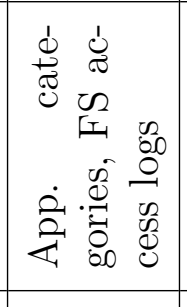 & 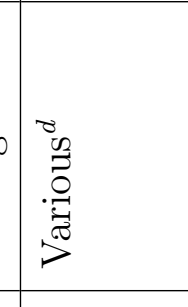 \\
\hline 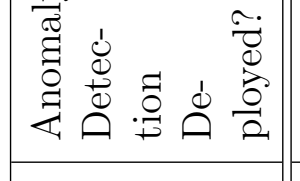 & 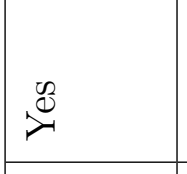 & $\stackrel{8}{2}$ & 里 & 8 & \& \\
\hline 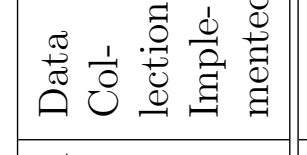 & $\stackrel{\infty}{\circ}$ & $\stackrel{\mathscr{D}}{*}$ & 号 & 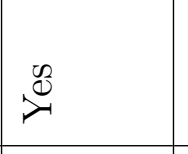 & 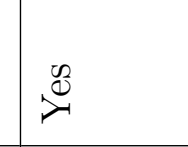 \\
\hline 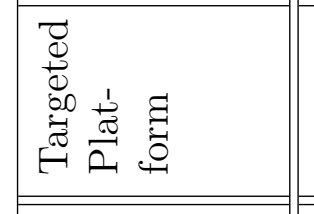 & 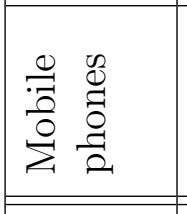 & 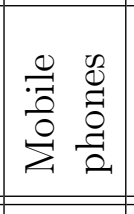 & 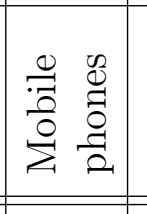 & 禹 & 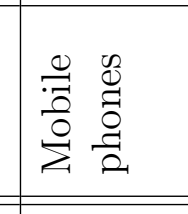 \\
\hline 吾 & 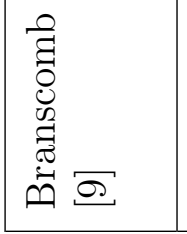 & 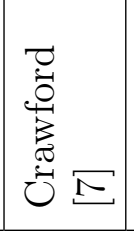 & $\begin{array}{l}\exists \\
ت \\
0 \\
ت \\
ت\end{array}$ & 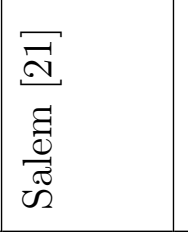 & 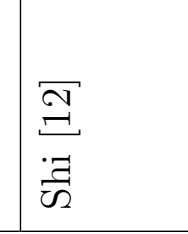 \\
\hline
\end{tabular}

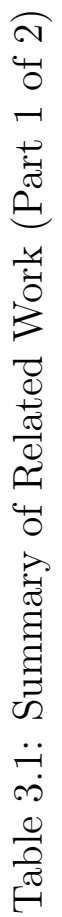

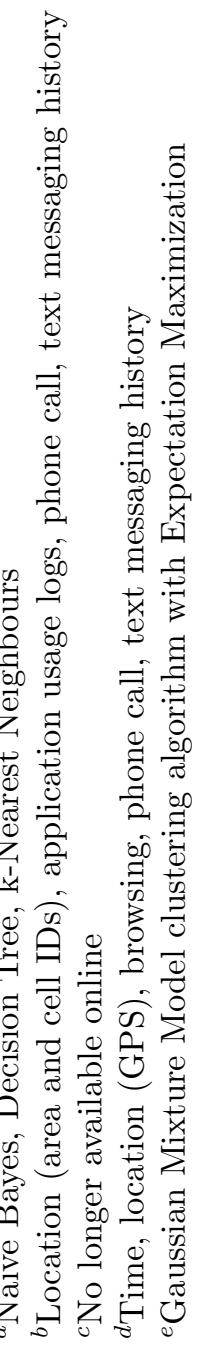




\begin{tabular}{|c|c|c|c|c|}
\hline 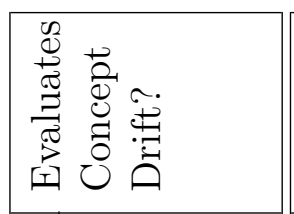 & $\stackrel{8}{2}$ & 号 & \&̊ & 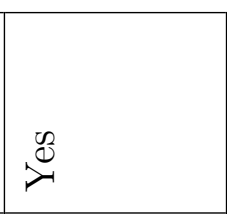 \\
\hline 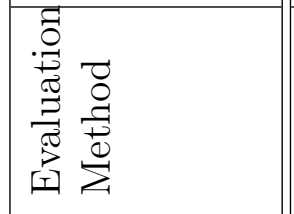 & 密 & 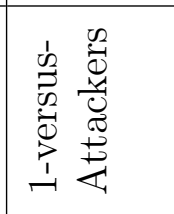 & 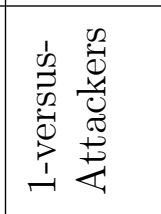 & 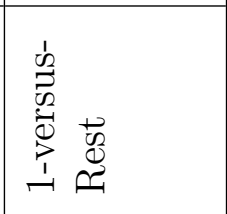 \\
\hline 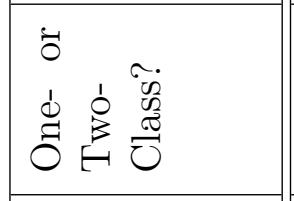 & $\mathscr{\Xi}$ & $\Xi$ & $\Xi$ & $\Xi$ \\
\hline 意 & 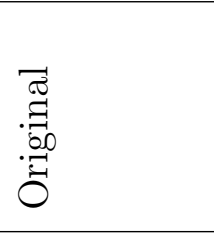 & 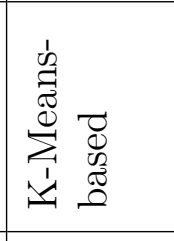 & 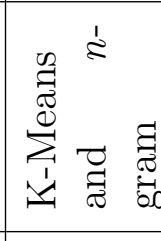 & 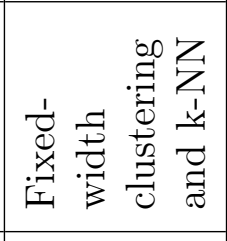 \\
\hline & 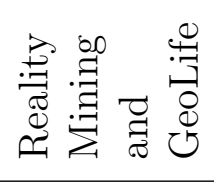 & 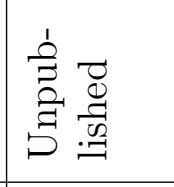 & 言营 & 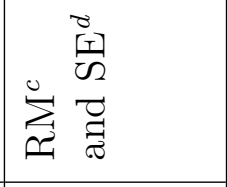 \\
\hline & $\stackrel{8}{z}$ & 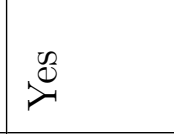 & 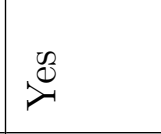 & 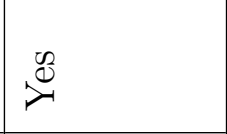 \\
\hline & 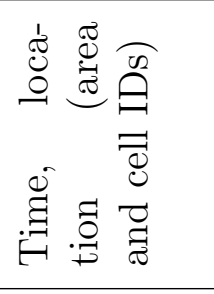 & 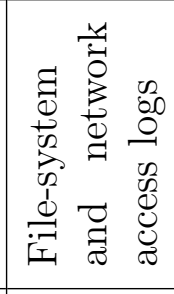 & 童 & 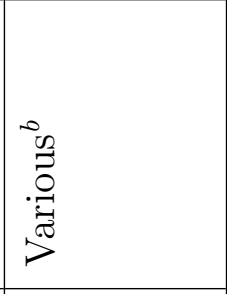 \\
\hline : & $\stackrel{8}{2}$ & 号 & 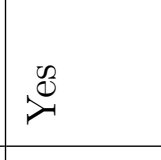 & 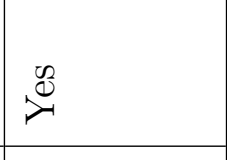 \\
\hline $\bar{Q}$ & $\stackrel{8}{2}$ & $\stackrel{\mathscr{D}}{\nearrow}$ & $\stackrel{\mathscr{B}}{:}$ & 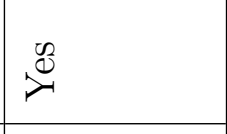 \\
\hline 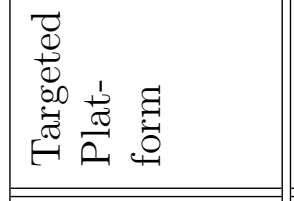 & 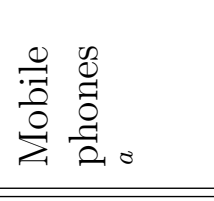 & 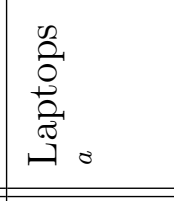 & 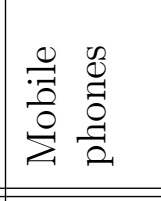 & 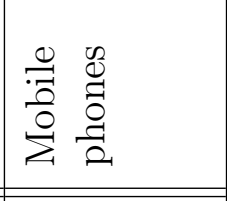 \\
\hline 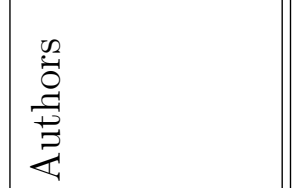 & : & 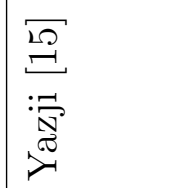 & 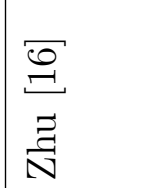 & 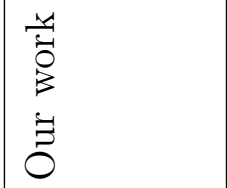 \\
\hline
\end{tabular}

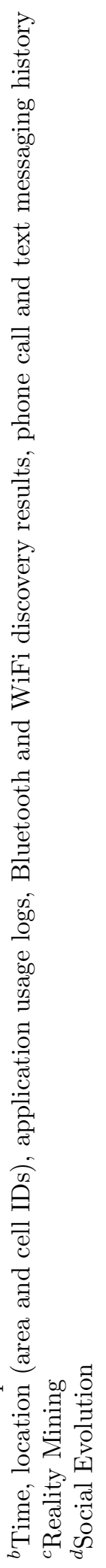




\section{Chapter 4}

\section{Methodology}

In this chapter, we introduce our methodology. We first describe the two data sets we use for evaluation, the features they include, and their limitations. Afterwards, we describe how the time slice model can be used to summarize a mobile phone user's behaviour, with which we perform anomaly detection. The chapter ends with a brief discussion of the application of match score level fusion, with which we experiment in an effort to overcome difficulties encountered with our default method of feature level fusion.

\subsection{Data Sets}

We make use of the "Reality Mining" and the "Social Evolution" data sets, which were made available by the MIT Media Lab's Human Dynamics Group. [19,22]

The Reality Mining data set is the result of a study between September 2004 and June 2005, in which 95 subjects, the 68 of whom were graduate students or staff of the MIT Media Lab, and the remaining of whom were new students at MIT's business school. The subjects were given Nokia 6600 smartphones, which had a logging application installed, for the duration of the study. The logging application logged a variety of data made available by the phone's software, which includes detailed logs of 
communication events (incoming, outgoing and missed calls, and text messages), the location of the mobile phone as inferred from the area and cell identifiers of cellular towers, whether the phone was in use or not, whether the phone was being charged or not, names of the applications which were started by the user, and the results of periodic (where the period length is 5 minutes) Bluetooth scans. The logged data was timestamped.

The Social Evolution data set is the result of a study between October 2008 and May 2009, where roughly 80 students staying in the same dormitory at MIT installed data collection software on their mobile phones. For each user, the collected data includes periodic Bluetooth scan results, MAC addresses of Wi-Fi access points which were discovered as a result of periodic Wi-Fi scans, and the hashed phone numbers to which phone calls were made and with which text messages were exchanged. The Bluetooth and Wi-Fi features were recorded every 6 minutes.

\subsection{Data Set Limitations}

One limitation of the Social Evolution data set involves the use of user identifiers instead of Bluetooth addresses. Whenever a mobile phone belonging to a study participant is detected by another participant, the detected user's user identifier is recorded. (For example, if users 1 and 2 are within a short distance for more than 6 minutes, then each user's mobile phone records the user identifier of the other user.) This limitation leads to another one: Out of all of the discovered Bluetooth devices, only the ones belonging to the participants of the study are recorded. We should note that the above-mentioned limitations do not exist with the Reality Mining data set.

Another Social Evolution data set limitation is that only outgoing phone calls are logged.

In contrast, the Reality Mining data set, because of its collection time, may not 
fully represent today's mobile phone usage characteristics. One example is the fact that with the relatively recent market penetration of smartphones with Android and iOS operating systems, the number of applications available for installation on smartphones has increased since 2004. Li et al. discuss this aspect of the data set in [10] as well. While what we mention is a limitation, our methods may have the potential of being more powerful should they be deployed on current mobile platforms.

Finally, another limitation of the Reality Mining data set is that the location information (which is in the form of area and cell IDs of the cellular tower to which the mobile phone is connected) is recorded only when the mobile device switches cellular towers. As we will note below, for our purposes, this required preprocessing the location feature in the Reality Mining data set.

\subsection{Overview of the Features Available in the Data Sets}

In this section we provide a more detailed overview of the features in the two data sets.

\subsubsection{Reality Mining Data Set}

The Reality Mining data set contains various features; we note here the ones which are most relevant to our study.

Communication Event Logs The communication event logs encompass the following events: phone calls, text messages and packet data sessions. The following features are recorded: timestamp, a unique event identifier, the contacts list index of the other party's phone number if the other party is in the contacts list (or -1 otherwise), the direction of the communication event (incoming, outgoing, missed), 
the duration of the communication event, and finally, the hashed phone number of the other party.

Location As mentioned before, the location of the mobile phone is recorded in the form of (timestamp, area ID, cell ID) tuples, which are recorded only when the mobile phone switches to another cellular tower.

Bluetooth Discovery Results For each study participant's mobile device, the following features are recorded with each Bluetooth discovery, which occurs every 5 minutes: the Bluetooth (MAC) address, the human-readable name, and the device type of the devices which were detected, and a timestamp.

Application Usage The application usage is logged in the form of (timestamp, name of the started application) tuples. Another table also contains the total number of times each application was used by the user (which we assume refers to the whole duration of the study).

Phone Status The data set contains three phone status features, which are whether the phone is actively being used or not, when the phone is switched on or off, and whether the phone is being charged or not. These events are timestamped. We assume that the timestamps and the statuses are recorded when the phone's status changes.

\subsubsection{Social Evolution Data Set}

Bluetooth Discovery Results For each Bluetooth discovery made by each user's mobile device, the following attributes are recorded: identifier of the user whose mobile phone scanned for nearby Bluetooth devices, timestamp, the user identifiers of the owners of the detected Bluetooth devices, and finally, a probability value, 
(between zero and one), which indicates whether the users were on the same floor, and which was added to the data set after the experiment by the collectors of the data set.

Wi-Fi Discovery Results Similarly, for each Wi-Fi discovery made by each user's mobile device, the following attributes are recorded: identifier of the user whose mobile phone scanned for Wi-Fi access points, timestamp, the hashed MAC addresses of the discovered access points, and the signal strength of each discovered access point.

Phone Call Logs The data set makes available the following attributes for each phone call that is made by a user: identifier of the user making the phone call, timestamp, duration of the phone call, (if the user is part of the study, then) the other party's user identifier, and finally, a hashed version of the phone number which was called.

Text Message Exchange Logs The data set makes available the following attributes for each text message that is sent by a user: identifier of the user sending a text message, timestamp, whether the text message was incoming or outgoing, (if the user is part of the study, then) the other party's user identifier, and finally, a hashed version of the phone number with which a text message was exchanged.

\subsection{Feature Selection}

For each user, we select the following features out of the ones listed above.

\subsubsection{Reality Mining Data Set}

- Phone Call Logs: the hashed phone number of the other party, 
- Text Message Exchange Logs: the hashed phone number of the other party, and whether the text message was incoming or outgoing,

- Location: area and cell identifiers of the cellular tower,

- Bluetooth Discovery Results: the discovered devices' Bluetooth (MAC) addresses and names,

- Application Usage Logs: the names of the applications which are used.

\subsubsection{Social Evolution Data Set}

- Bluetooth Discovery Results: the discovered users' identifiers (in "fake" Bluetooth address form),

- Wi-Fi Discovery Results: the MAC address hash in MAC address form,

- Phone Call Logs: the hashed phone number of the other party,

- Text Message Exchange Logs: the hashed phone number of the other party, and whether the text message was incoming or outgoing.

\subsection{Preprocessing of the Reality Mining Data Set}

As was mentioned above, the location feature in the Reality Mining data set was recorded in the form of (timestamp, area ID, cell ID) tuples, and these tuples were recorded only when a cellular tower transition was made by the study participant's mobile device.

During our work, we found out that there exists another version of the Reality Mining data set which includes the amount of time spanned by each cellular tower connection of the participants' mobile devices; however, this version of the Reality Mining data set is more limited in terms of the number of features compared to the version that we are using.

In [23], Bayir et al. report that in the above-mentioned version of the data set 
with cellular tower connection time spans, $94 \%$ of the cellular tower connections last for less than 10 minutes.

Because of difficulties in cross-referencing the two versions of the data sets, we make use of Bayir et al.'s findings to extrapolate the study participants' locations for at most 10 minutes. For example, if there is a location recorded for a study participant, but however, no locations are recorded for up to 10 minutes, we assume that the last location of the user had been valid for 10 minutes. Using this method, we aim to fill in the discontinuities in the data set's location feature.

\subsection{Data Model}

Both data sets are composed of timestamped feature type and feature value pairs. As such, we represent data points as:

$$
\left(\text { feature }_{i x}\right)_{u, \text { date }, t}
$$

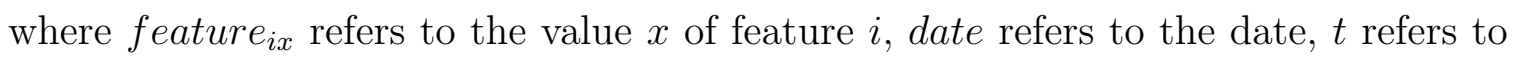
the time of day, and $u$ refers to the identifier of the user.

Therefore, we represent a data set as follows:

$$
\left\{\left(\text { feature }_{i x}\right)_{u, \text { date,time }} \quad \forall u \in\left(u_{1}, u_{2}, \ldots, u_{\text {num_users }}\right)\right\}
$$

Out of the data sets, for each user, we consider each day during the study in which the user in question has generated some data. We divide each day into equal-length "time slices," each of which contains a "bag" of features that occur during the time slice in question.

We define time slices of length time_slice_length, which are indexed with $k$ for a 
day specified by date in the user $u$ 's data, as follows:

$$
\begin{aligned}
\left\{\left(t_{\text {start }}, t_{\text {end }}, \text { featureBag }\right)_{u, \text { date }, k}\right. & \mid \\
& \left(t_{\text {end }}-t_{\text {start }}=\text { time_slice_length }\right) \wedge \wedge \\
& \left(\left(t_{\text {start }}\right)_{u, \text { date }, 1}=00: 00: 00\right) \wedge \\
& \left(\left(t_{\text {end }}\right)_{u, \text { date }, k_{\max }}=23: 59: 59\right) \wedge \\
& (\text { featureBag })_{u, \text { date }, k}= \\
& \left.\left\{(\text { feature })_{\text {ix }}\right)_{u, \text { date }, t} \mid\left(t \geq t_{\text {start }}\right) \wedge\left(t \leq t_{\text {end }}\right)\right\} \wedge \\
& \mid\left(\text { featureBag }_{u, \text { date }, k} \mid>0\right\}
\end{aligned}
$$

The above was a formal definition of our data model. In our implementation, instead using a featureBag, we use a dictionary for each feature type $i$. The keys of the dictionaries are the feature values $x$, and the values of the dictionaries are the number of occurrences of the value feature $_{i x}$ during the time slice in question. This way, we count, for example, how many phone calls to a specific number were made in each time slice.

There is only one exception to this counting scheme: For the cellular location feature type, we record the number of minutes that were spent in each cellular location identifier during the time slice.

As an example, suppose user $u$ has the following features in his/her part of the data set:

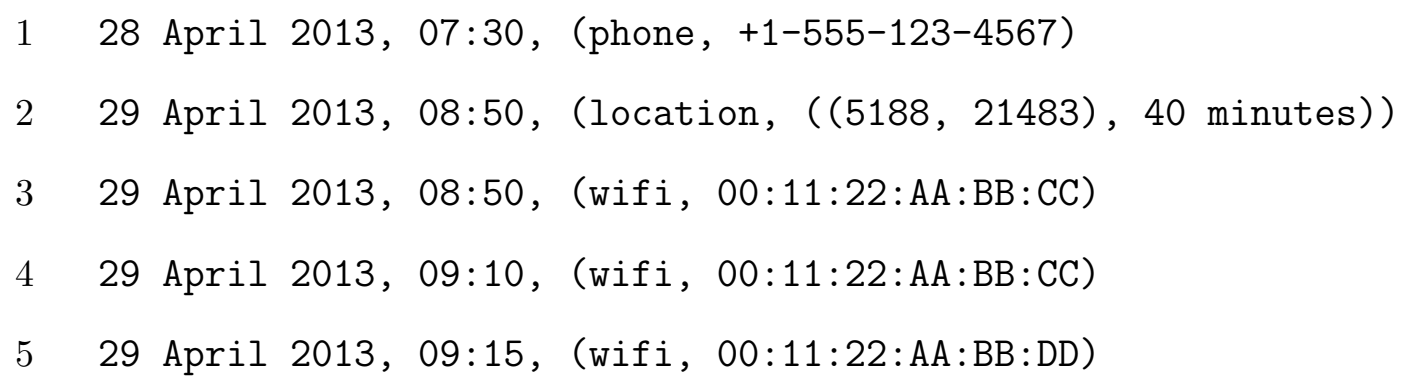


If we choose the time slice length $N$ to be 8 hours, then two time slice data structures will be instantiated for user $u$, which can be seen in Figure 4.1: One for the first 8-hour-long time slice of April 28th, 2013, and another one for the second 8-hour-long time slice on April 29th, 2013.

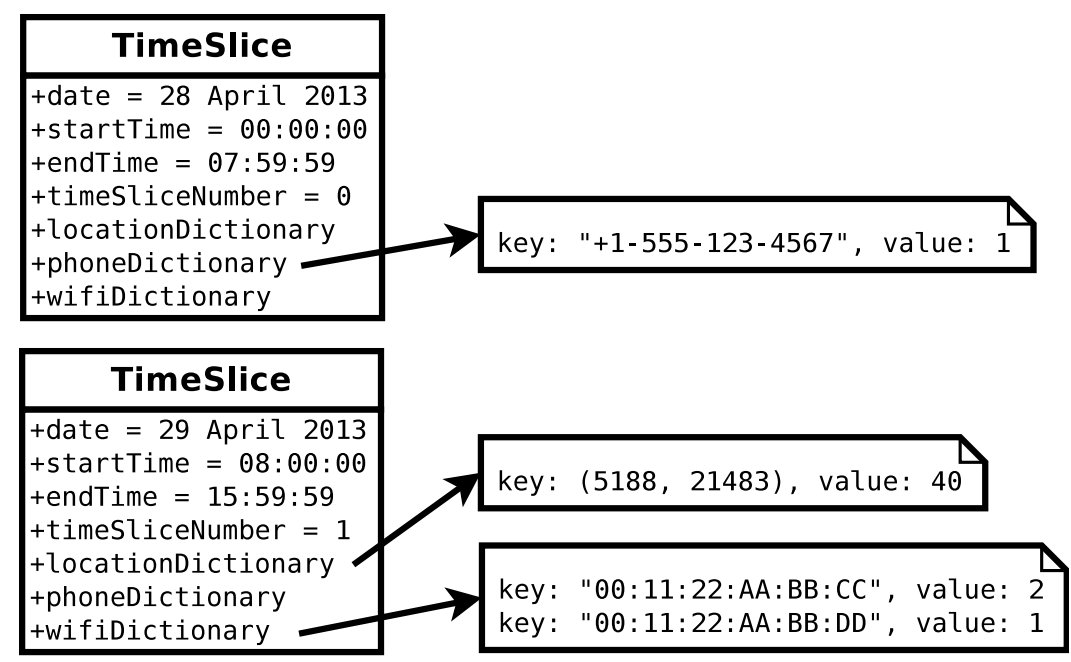

Figure 4.1: Two examples of time slice data structures.

As can be seen in Figure 4.1, the time slice corresponding to the first time slice of April 28th, 2013 will have only the dictionary corresponding to "phone" nonempty. The second time slice of April 29th, 2013 will have two non-empty dictionaries: "location," and "wifi." Note that the "wifi" dictionary will have a value of 2 for the key "00:11:22:AA:BB:CC", as that Wi-Fi access point MAC address had been observed twice during the time slice. Similarly, because the mobile device had been in the location identified with the area and cell identifier tuple "(5188, 21483)" for 40 minutes, we record 40 as the value of the key formed by that area and cell identifier tuple.

\subsubsection{Regarding "Empty" Time Slices}

As we have noted above in this section, we instantiate time slices only for the days during which the data set participant has "generated some data," by making a phone 
call, or changing his/her location, for example. Furthermore, as noted in Section 4.3.1, the Reality Mining data set includes mobile phone status features, one of which indicates whether a participant's mobile phone is on or off.

While examining the Reality Mining data set, we noticed the existence of "empty" time slices, which are time slices during which no events (such as location changes or outgoing phone calls) take place, but the mobile phone is switched on.

Even though the lack of events during a time slice which is usually "non-empty" can be considered an anomaly, for almost all of our experiments we ignore empty time slices, mainly because we do not expect a mobile phone to authenticate a user when no data is present "now."

\subsection{Distance Function}

We use two of the three anomaly detection algorithms which were introduced by Eskin et al. in [3], a summary of which was provided in Section 2.2: fixed-width clustering, and an optimized variant of k-Nearest Neighbours, which makes use of fixed-width clustering.

Both of these anomaly detection algorithms utilize a distance/comparison function which is used to determine the distance between two data points. In our case, the data points are the data structures that were instantiated for each user as explained in the previous section.

To compare two data points in the data sets, we use the distance function shown in Algorithm 1.

As can be seen in Algorithm 1, if the two data structures represent time slices that are more than leniency_threshold amount of time apart from each other, then we return the maximum distance, which is 1.0. The leniency threshold is used to find out whether we can exploit cyclical behaviours of the users by forcing the anomaly 


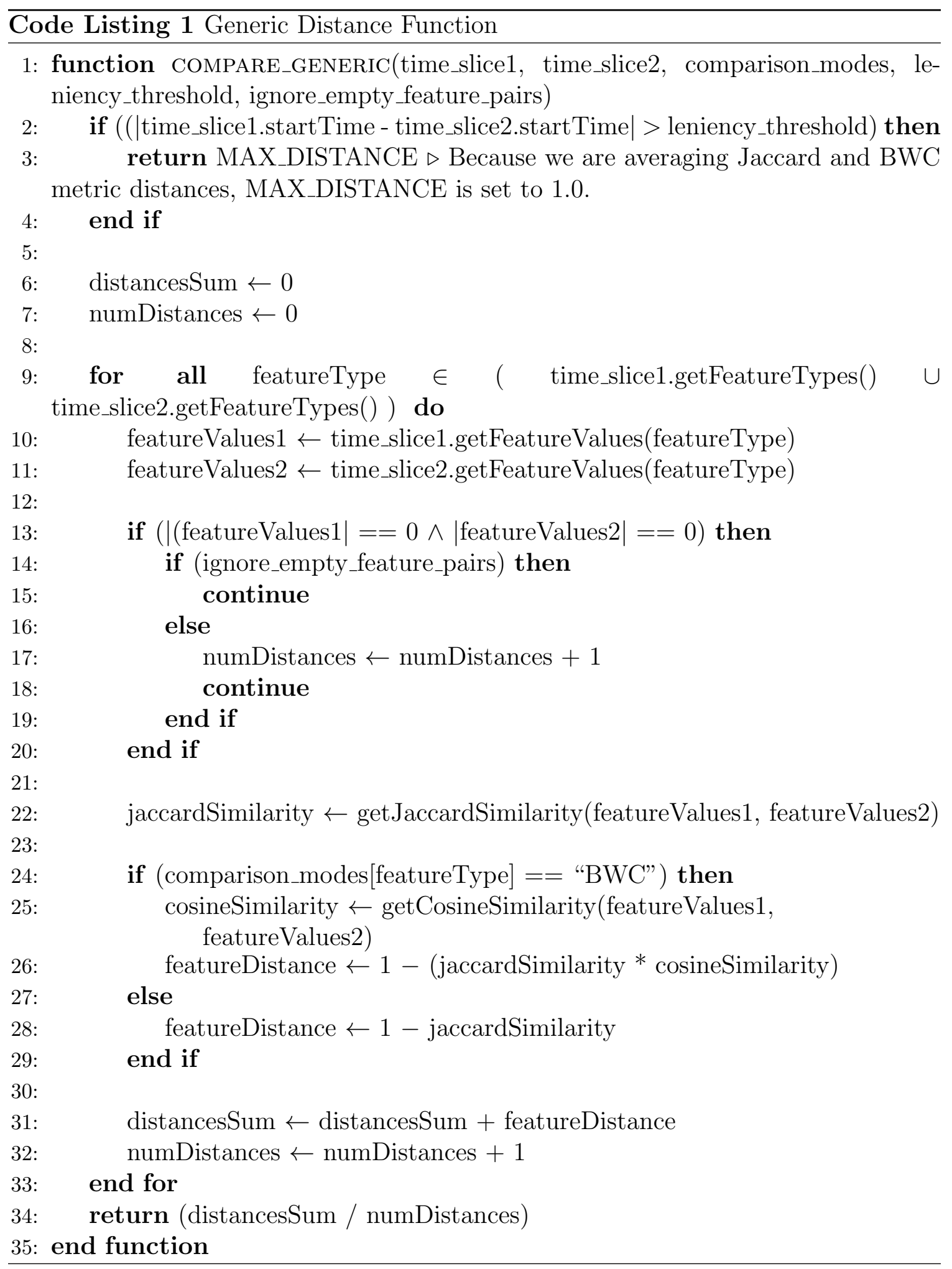


detection algorithm to never cluster together or consider as near neighbours the time slice data structures which do not occur at approximately the same time of the day.

Before we explain the remaining part of the distance function, we would like to note that to compare two feature dictionaries, we use one of the following distance metrics:

\section{- Jaccard Distance}

This is a distance metric used to calculate the amount of non-overlap between two sets. Formally, the definition of the Jaccard distance between set A and set $\mathrm{B}$ is as follows:

$$
\begin{aligned}
\text { jaccardSimilarity } & =\frac{|A \cap B|}{|A \cup B|} \\
\text { jaccardDistance } & =1-\text { jaccardSimilarity }
\end{aligned}
$$

This distance metric's values range between 0 and 1 , inclusive, where sets with more overlap have distances closer to zero.

- Binary Weighted Cosine (BWC) Distance

This distance metric was obtained from [24]. It is based on the Jaccard similarity and the cosine similarity metrics, and it operates on bags of items as opposed to sets of items.

The formal definition of the metric is as follows:

$$
\begin{aligned}
\text { jaccardSimilarity } & =\frac{|\operatorname{set}(A) \cap \operatorname{set}(B)|}{|\operatorname{set}(A) \cup \operatorname{set}(B)|} \\
\text { cosineSimilarity } & =\frac{\operatorname{vector}(A) \cdot \operatorname{vector}(B)}{\| \text { vector }(A)\|*\| \text { vector }(B) \|} \\
\text { bwcSimilarity } & =\text { jaccardSimilarity } * \text { cosineSimilarity } \\
\text { bwcDistance } & =1-\text { bwcSimilarity }
\end{aligned}
$$


where the function set converts the bags into sets, and the function vector converts the bags into integer vectors, the elements of which indicate the number of occurrences of each value in the bag, with the extension that the items which do not exist in one bag $A$ but exist in bag $B$ are expressed as having counts of zero in the vector form of $A$ and vice versa. The vectors have the same order with respect to the items of whose counts each element represents.

This distance metric not only considers the overlap between two bags, but also the "strength" of the overlap in terms of the counts of items in the bags. This metric also produces distance values between 0 and 1 , inclusive.

As can be seen on lines $22-29$ of Algorithm 1, features can be compared using either the Jaccard or the BWC distances, which we have introduced above. The dictionary comparison_modes controls which features are compared using which distance metrics.

The implication of using the Jaccard or the BWC distance metric is the following: If we consider the phone call feature, for example, with the Jaccard distance, we consider only which phone numbers had been called in the two time slices being compared. With the BWC distance metric, we consider not only which phone numbers had been called, but also the number of times each phone number in the two time slices had been called.

Finally, on line 34, the function returns the average of the Jaccard or BWC distances as the distance between the two data structures, which is always between zero and one. Using the average of Jaccard and BWC distances lets us assign equal weight to all available features in the two data structures. We should note that a weighted sum scheme is certainly possible.

One open-ended problem regarding the distance calculation is how unavailable features, that is, features for which both of the time slices being compared have empty dictionaries, should be considered. We make available two options, which 
are controlled using the ignore_empty_feature_pairs flag. If this flag is set, then unavailable features are simply excluded from the distance calculations, as shown on lines 14 - 16. If not, then two empty dictionaries are considered "equal" and we effectively add an entry with a value of zero to the averaged distances, as seen on lines $16-18$.

Based on preliminary experiments showing that taking into account empty feature pairs reduces the accuracy of our approach, we always keep this option enabled - that is, we always ignore empty feature pairs.

\subsection{Fusion}

As was discussed in Section 2.4, there are three approaches to fusion in biometrics: feature level, match score level, and decision level.

The distance function in Algorithm 1 makes use of feature level fusion because of the way multiple features are considered as one larger feature in the form of a time slice.

In this section, we discuss another fusion method that we evaluate in this document.

\subsubsection{Motivation for Match Score Level Fusion}

Our intuition in using more features has been that the use of larger number of features would help us verify the identity of a mobile device user more accurately. ${ }^{1}$

While conducting experiments, we noticed that the averaging aspect of the distance function had unexpected consequences. For example, suppose that we use only the phone call feature in an experiment, and we obtain a performance measurement in

\footnotetext{
${ }^{1}$ Given the relatively small number features we had, we did not find the "curse of dimensionality" very probable in our application.
} 
the form of an $\mathrm{AUC}$ value equal to $A U C_{\text {phone. }}$ Similarly, suppose that an experiment with only the Bluetooth feature produces an $\mathrm{AUC}$ value equal to $A U C_{B l u e t o o t h}$, where $A U C_{\text {Bluetooth }}<A U C_{\text {phone, }}$ indicating that the Bluetooth feature has less predictive power in verifying the identity of a mobile device user in the data set on which we are running the experiments.

During the evaluation of our methodology, if we were to run the same experiment with the phone and the Bluetooth features, then we saw that the resulting AUC performance metric value would be between $A U C_{\text {phone }}$ and $A U C_{\text {Bluetooth }}$ as opposed to being higher than $A U C_{\text {phone }}$.

In an attempt to solve this issue, in addition to feature level fusion, we implement match score level fusion, based on the intuition that there can be an "expert" anomaly detection model for each feature, and the combination of these anomaly detection models may achieve performance that is better than the performance resulting from the best individual feature.

\subsubsection{Match Score Level Fusion Methodology}

As mentioned above, to implement match score level fusion, we instantiate and train a separate anomaly detection model for each feature, and afterwards, during the testing phase, we combine the "anomaly scores" produced by the individual anomaly detection models to obtain a single anomaly score for each time slice under consideration. Finally, using the combined anomaly score, a prediction (such as "normal" or "attack") can be made.

To implement the above-mentioned approach, we represent each time slice as a composition of child data structures, each of which represents a feature dictionary which we discussed in Section 4.6. The child data structures representing the individual feature dictionaries are then used to train an anomaly detection model specific to their feature type. 
In terms of the distance function used to compare two child data structures, we use a distance function similar to the one in Algorithm 1, with the modification that we compare only two dictionaries that have times of day attached. When comparing the dictionaries, we can use either the Jaccard or the BWC distance metric.

To combine the anomaly scores resulting from the feature-specific anomaly detection models, we experiment with two methods, the first one of which is discussed by Jain et al. in [25]: "simple sum" and "match score-based voting." The simple sum score method combines individual anomaly scores by taking their sum, whereas the match score-based voting method uses a threshold value to let each anomaly score resulting from a different feature type to vote "attack" or "normal," based on whether an anomaly score is above or below the threshold value.

\subsection{Conclusion}

In this chapter, we have introduced the Reality Mining and the Social Evolution data sets and their respective features that we use for the evaluation, and we have discussed the time slice model, which can be implemented with feature level fusion and match score level fusion methods, which, as will be seen in the next chapter, have different performance characteristics. 


\section{Chapter 5}

\section{Evaluation}

In this chapter, we evaluate the time slice model in conjunction with Eskin et al.'s fixed-width clustering and k-NN-based anomaly detection algorithms using the Reality Mining and Social Evolution data sets. While carrying out this task, we also investigate the contributions of individual features to the anomaly detection accuracy.

The general methodology of our evaluation experiments is as follows: For a particular data set, we consider each participant of the data set the "owner" of a mobile device, and determine whether the one-class machine learning approach we use is able to detect the other participants of the data set as attackers. Using the terminology of the Related Work chapter, our approach is a 1-versus-Rest approach.

A two-class method can be considered as a "supervised anomaly detection" approach, which had been discussed in Section 2.1. The disadvantage of such an approach would be the need to maintain a labeled data set containing attacker/positive behavioural patterns deployed with the system. In contrast, a one-class ("semi-supervised") approach can make use of only the negative (that is, normal) behaviours of the user/owner of the mobile device and detect deviations from the

owner's behavioural profile. For these reasons, we believe a one-class approach is more appropriate in our application domain. 


\subsection{Dynamic User Profiles}

We use dynamic user profiles so that we can train a classifier based on only the $N$

days before "today," thereby focusing on the most recent data which represents the mobile device user's behaviour.

We consider each participant of the data set as the "owner" of a mobile device, one at a time. We use a sliding window approach, where the current "owner" participant's data is considered a stream. As an example, we start by training a one-class classifier on the first $N$ days of the "owner" participant's data, where $N$ can be varied. Afterwards, we test the one-class classifier on the owner's $(N+1)$ th day's negative example containing data plus a multi-stage cluster sample (which is explained below) of all of the "attacker" participants' positive example containing data.

In the next "iteration" of the evaluation, we shift the training period to the right by one day, and test on the $(N+2)$ th day's data and another multi-stage cluster sample of all of the "attacker" participants' data.

We call all of the iterations of one owner participant's evaluation a sub-experiment. An experiment, therefore, is the collection of all of the iterations from all of the possible owner participants.

We illustrate our method with Figures 5.1a and 5.1b.

\subsection{Multi-Stage Cluster Sampling}

As was mentioned above, we use multi-stage cluster sampling to sample a number of time slices from the attacker participants' data.

With multi-stage cluster sampling, it is possible to give various already-existing distributions in the data being sampled an equal priority. As an example, consider a city, from which we would like to sample a number of households, which we would 


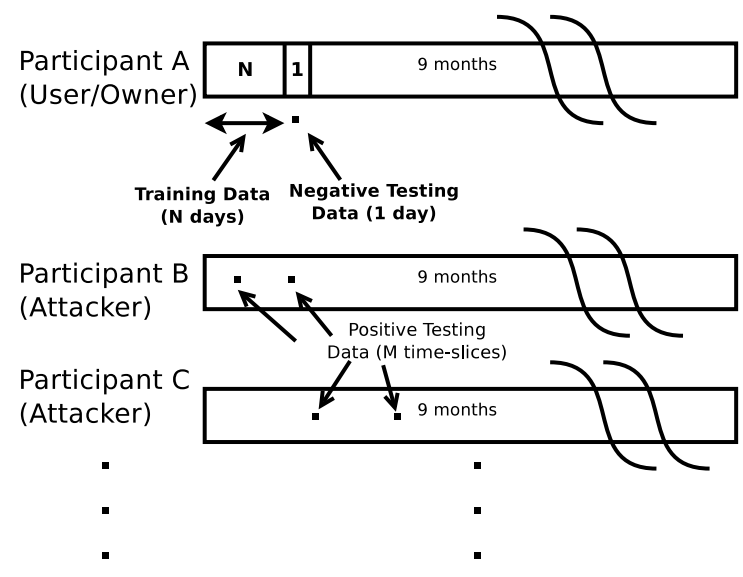

(a) First Iteration of a Sub-experiment

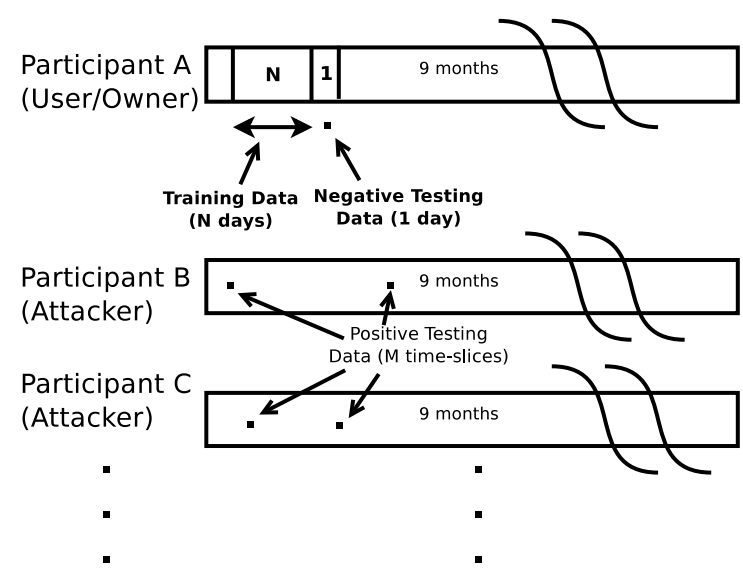

(b) Second Iteration of a Sub-experiment

Figure 5.1: Illustration of a Dynamic User Profile Sub-experiment. In Figure 5.1a, a classifier is trained on the first $N$ days' data and tested with the $(N+1)$ th day's data and a sample of the attacker participants' data. For the second iteration, which is illustrated in Figure 5.1b, we shift the training period and the negative testing day to the right by one day, and take a new sample of positive time slices from the attacker participants' data.

like to survey, from all of the households in the city. We can first divide the city into 4 suburbs, and divide each suburb into a number of blocks. Afterwards, we can sample a number of families from a number of blocks from a number of suburbs.

In our case, the suburbs are made up of the time slice categories, where each category represents a different time of day, and blocks are made up of different attackers, and finally, the individual families are the individual time slices.

Using this approach, we can assign equal probability of choosing a time slice belonging to $4 \mathrm{am}$ from user $X$ to choosing a time slice belonging to $8 \mathrm{pm}$ from user $Y$. This way, we represent all attackers and all times of the day equally while sampling.

\subsection{Important Notes}

There are two important aspects of this approach to note regarding the testing phase:

1. There is a large class imbalance, where the negative examples are only from one 
day of the owner's data, whereas the number of the positive examples can be as large as the size of the multi-stage cluster sample we take from the attacker participants' data.

To avoid having our results skewed by the class imbalance, we use AUC and EER as the classification accuracy metrics.

2. We use multi-stage cluster sampling to avoid very long experiment run-times. To let the reader have an appreciation of the time complexity of our experiments, let us note that there are 95 participants with data in the Reality Mining data set, with each participant having anywhere from 1 to 9 months' worth of data. Even when we consider 4 hour-long time slices, and assume that on average each participant has 60 days' worth of data with 6 time slices per day, we still need to train and test a one-class classifier $95 * 60=5700$ times per experiment, where, without sampling each test run takes (based on our empirical observations) approximately 6 to 7 minutes on a laptop computer with a mid-end Intel Core i5 processor.

\subsection{Effect of Multi-Stage Cluster Sampling on Per- formance}

To verify that the multi-stage cluster sampling method we apply to reduce the size of the positive testing sets does not significantly affect our results, we run two experiments with the Reality Mining data set - one with sampling and one without - and compare the results.

To avoid letting the experiments take a large amount of time, we randomly sample 15 Reality Mining data set participants to act as an owner one at a time, and we use the remaining participants from the whole data set as attackers. In other words, if 
there are $P$ participants in the whole Reality Mining data set, we test each of the sampled 15 owner participants against the remaining $P-1$ attacker participants.

In these two experiments, ${ }^{1}$ we use the k-Nearest Neighbours algorithm only, and we arbitrarily set $\mathrm{k}$ equal to 1 , the time slice length equal to 4 hours, the distance function time slice "leniency" equal to 8 hours, and the training period length equal to 21 days. The used features are application usage, Bluetooth discovery results, location (in the form of area and cell identifiers) and phone call and text messaging logs. All features were configured to use the Jaccard distance for comparison, except for the location feature, which was configured to use the BWC metric.

In the first experiment, we disable sampling of positive time slices. In the second experiment, we use multi-stage cluster sampling as follows: We use all of the time slice categories from all times of the day, and all of the attackers, and we sample 1 time slice from each combination of the time slice category and the attacker identifier. In other words, if we use the analogy from Section 5.2, we "go to" all of the suburbs of the city, all of the blocks of all of the suburbs, and interview one family from each suburb and block combination.

The results of the two experiments can be seen in Figure 5.2, where we present a scatter plot that shows how the AUC and the EER metric values produced by the two experiments' 1921 iterations vary. In the plots, the $y$ axis represents the AUC (or EER) metric values of the experiment without sampling, whereas the $x$ axis represents those of the experiment with sampling.

As can be seen from the figures, the distribution of the AUC and EER metric values of the experiment with sampling has a relatively strong linear relationship with those of the experiment without sampling.

To quantify the amount of error introduced by sampling, we plot histograms of the

\footnotetext{
${ }^{1}$ We should note that these two experiments were conducted with an older version of our software, which took into account empty time slices and ignored location identifier tuples equal to " $(0,0)$ ", which represent the times during which the mobile phone does not have signal.
} 


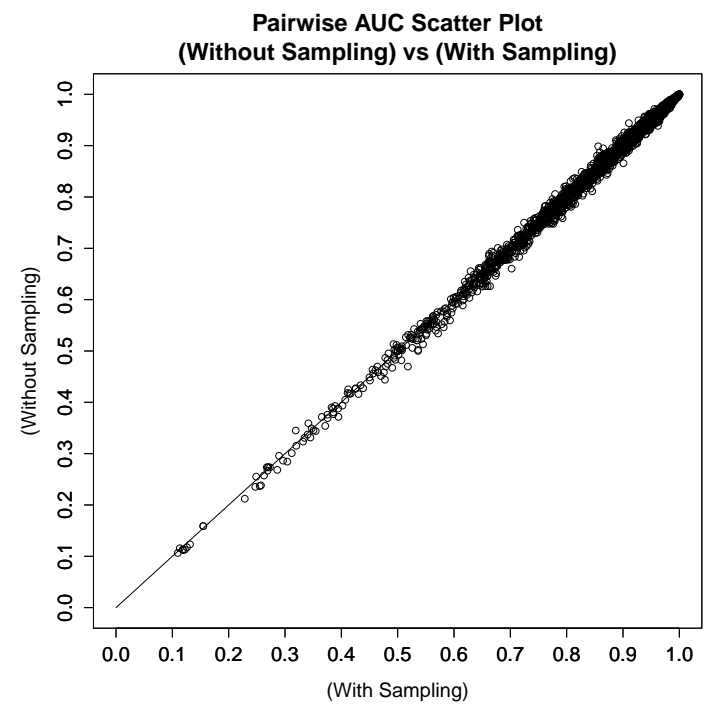

(a) Pairwise Scatter Plot Comparing the AUC Results of All Iterations of Experiments without Sampling ( $y$ Axis) and with Sampling ( $x$ Axis)

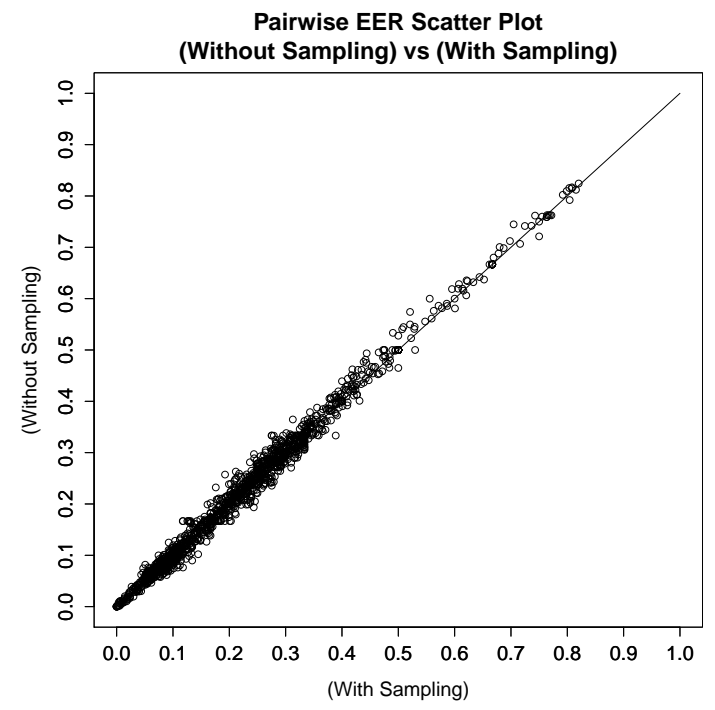

(b) Pairwise Scatter Plot Comparing the EER Results of All Iterations of Experiments without Sampling (y Axis) and with Sampling ( $x$ Axis)

Figure 5.2: Comparison of the Experiment Results with and without Multi-Stage Cluster Sampling

pairwise differences of the AUC and EER metric values resulting from the experiments with and without sampling. These plots are presented in Figure 5.3, where we can see that the majority of the pairwise AUC and EER differences are within \pm 1 percentage point. We should note, however, that - especially for EER - sampling makes the results appear slightly better.

In addition, we repeat the experiment making use of sampling, and increase the aggressiveness of the sampling used: Instead of selecting all 94 attackers in the multistage cluster sampling method, this time we sample only 20 attackers out of the 94 . For this experiment, we only present a histogram of pairwise deltas of AUC and EER metric values for brevity. The results can be seen in Figure 5.4, which indicates that the majority of the error introduced by more aggressive sampling is within \pm 2 percentage points.

Unless we note otherwise, we use the earlier moderate sampling approach with all 


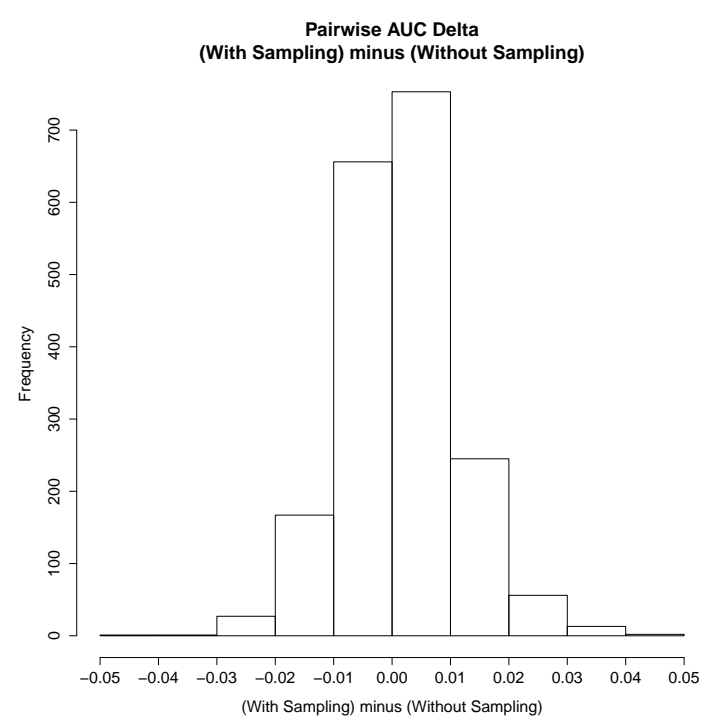

(a) Histogram of the Pairwise Delta between the AUC Results of All Iterations of Experiments with Sampling and without Sampling

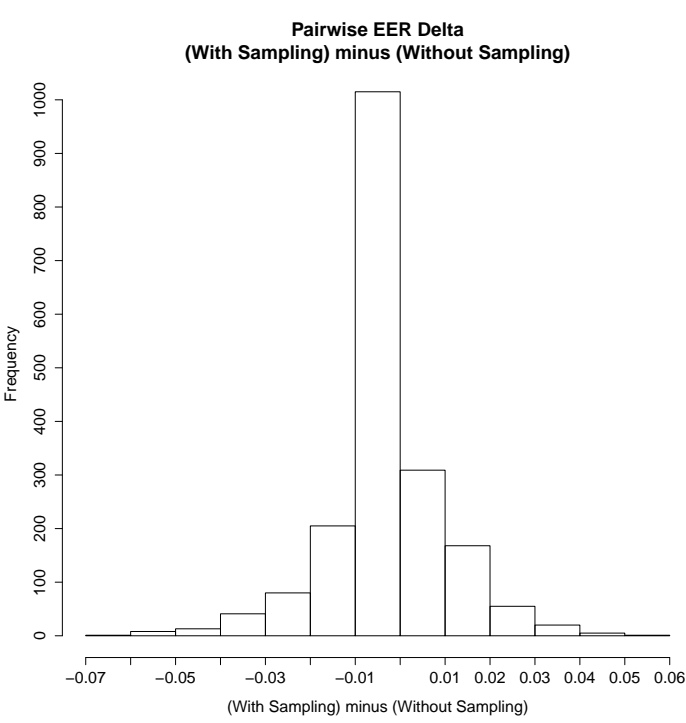

(b) Histogram of the Pairwise Delta between the EER Results of All Iterations of Experiments with Sampling and without Sampling

Figure 5.3: Comparison of the Experiment Results with and without Multi-Stage Cluster Sampling

of the following experiments.

\subsection{Effect of Classifier Parameters on Performance}

After confirming the acceptable non-effect of sampling on our evaluation strategy, we run experiments to find the parameters with which the fixed-width clustering algorithm and the k-Nearest Neighbours algorithm perform best. In addition, these experiments guide us in choosing the algorithm to use in the remaining experiments.

For k-Nearest Neighbours, we vary $k$ between 1 and 4 with a step size of 1 , and for fixed-width clustering we vary the cluster width between 0.3 and 0.9 with a step size of 0.1 .

For these experiments we use the Reality Mining data set only, and we use a time slice length and leniency of 4 hours, use all features, and compute the distances 


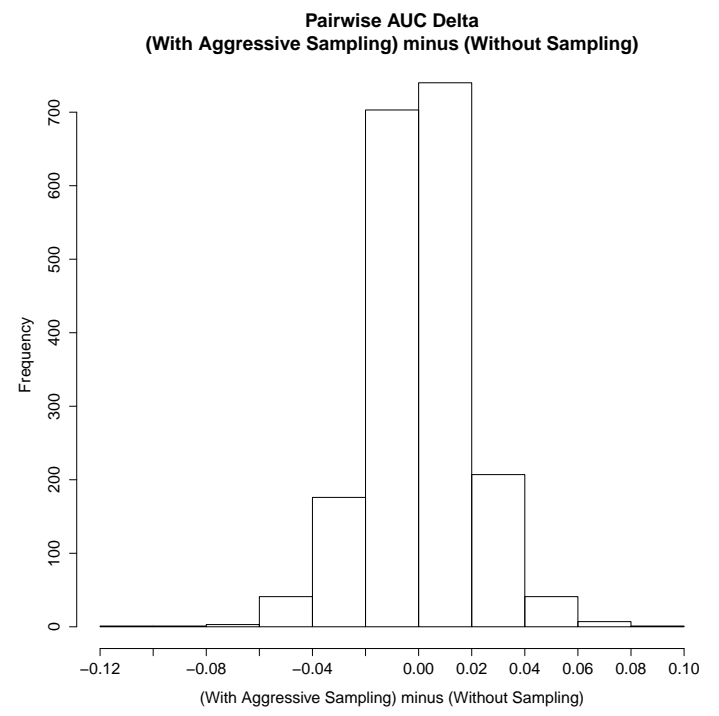

(a) Histogram of the Pairwise Delta between the AUC Results of All Iterations of Experiments with Aggressive Sampling and without Sampling

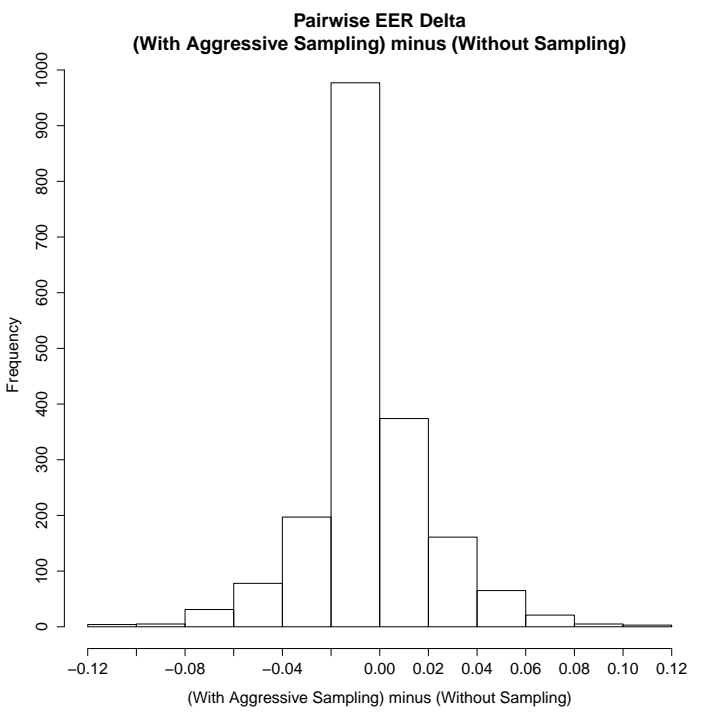

(b) Histogram of the Pairwise Delta between the EER Results of All Iterations of Experiments with Aggressive Sampling and without Sampling

Figure 5.4: Comparison of the Experiment Results with and without Aggressive Multi-Stage Cluster Sampling

between feature values using the Jaccard distance.

We present our results in terms of the distribution of AUC and EER performance metrics resulting from all iterations of the experiments in Figure 5.5, which contains boxplots of the distributions of the mentioned performance metrics.

As can be seen in the boxplots in Figure 5.5, while k-Nearest Neighbours has a better overall distribution of performance metrics for all experiment iterations, it is also burdened with a lot more outliers compared to the fixed-width clustering algorithm.

According to the boxplots, k-Nearest Neighbours is only slightly affected by the parameter $k$, whereas the fixed-width clustering algorithm is affected by the cluster width parameter in a visually appreciable fashion.

For k-Nearest Neighbours, we see that the distribution of performance metrics for all iterations is slightly tighter for $k=1$ compared to $k=4$. 
For the fixed-width clustering algorithm, we see that in terms of the overall mean of performance metrics, the configuration with $c w$ (that is, the cluster width) set to 0.7 performs better than other values of $c w$.

Based on these results, we decide to use k-Nearest Neighbours with $k=1$ for the remaining experiments.

\subsection{Classification Performance of Individual Fea- tures}

To have an understanding of the contributions of individual features to the classification accuracy, we run a set of experiments where we enable individual features one by one. Furthermore, we experiment with the Jaccard and the BWC distance metrics for each feature. For each experiment, we set $\mathrm{k}$ equal to 1 , the time slice length equal to 4 hours, the distance function time slice "leniency" equal to 4 hours, and the training period length equal to 21 days.

The results indicating the predictive power of the individual features in the Reality Mining data set and the Social Evolution data set are shown in the form of boxplots depicting the distribution of the AUC and EER metric values in Figures 5.6 and 5.7.

To provide an insight for the amount of data summarized by these boxplots, let us note that the Reality Mining data set involved 6644 iterations for text messaging feature, 10268 iterations for the Bluetooth feature, 10498 iterations for the phone call feature, 11247 iterations for the location feature, and 11472 iterations for the application feature. For the Social Evolution data set, there were 3144 iterations for the text messaging feature, 4286 iterations for the WiFi feature, 7630 iterations for the Bluetooth feature, and 8878 iterations for the phone call feature. 


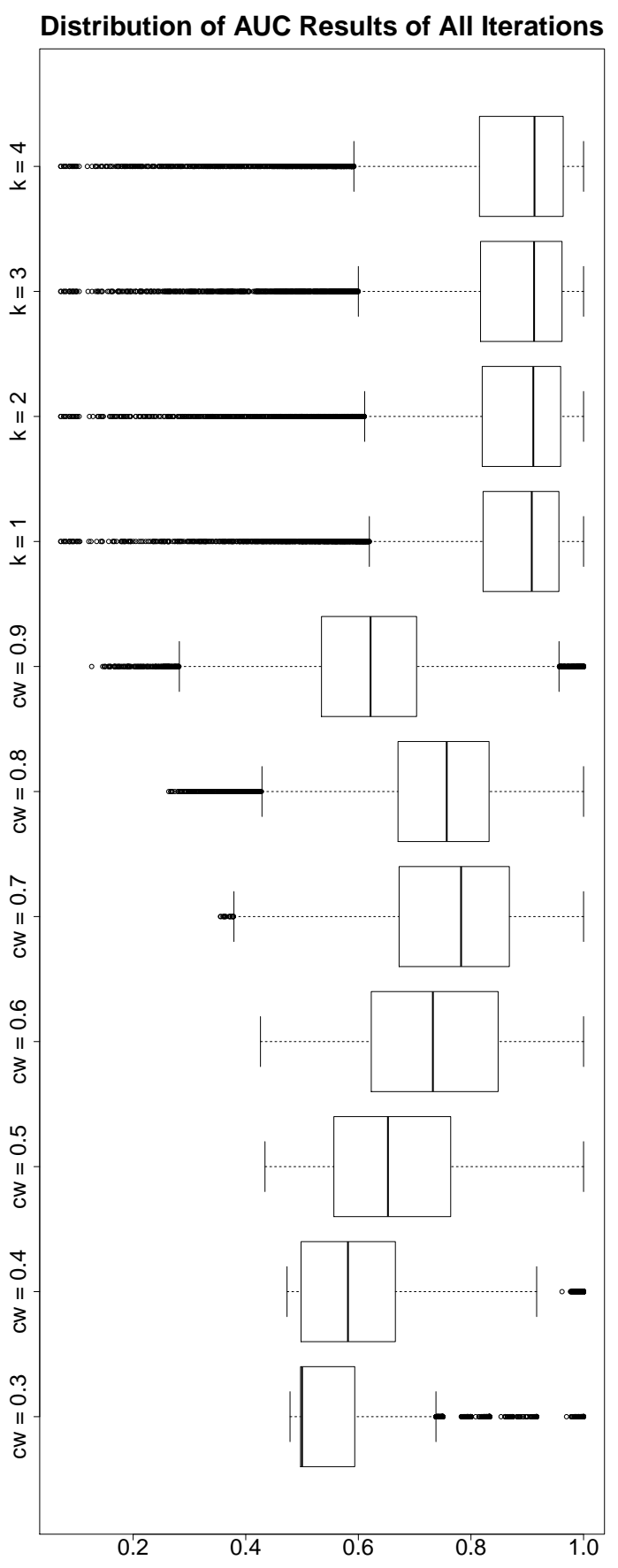

(a) AUC
Distribution of EER Results of All Iterations

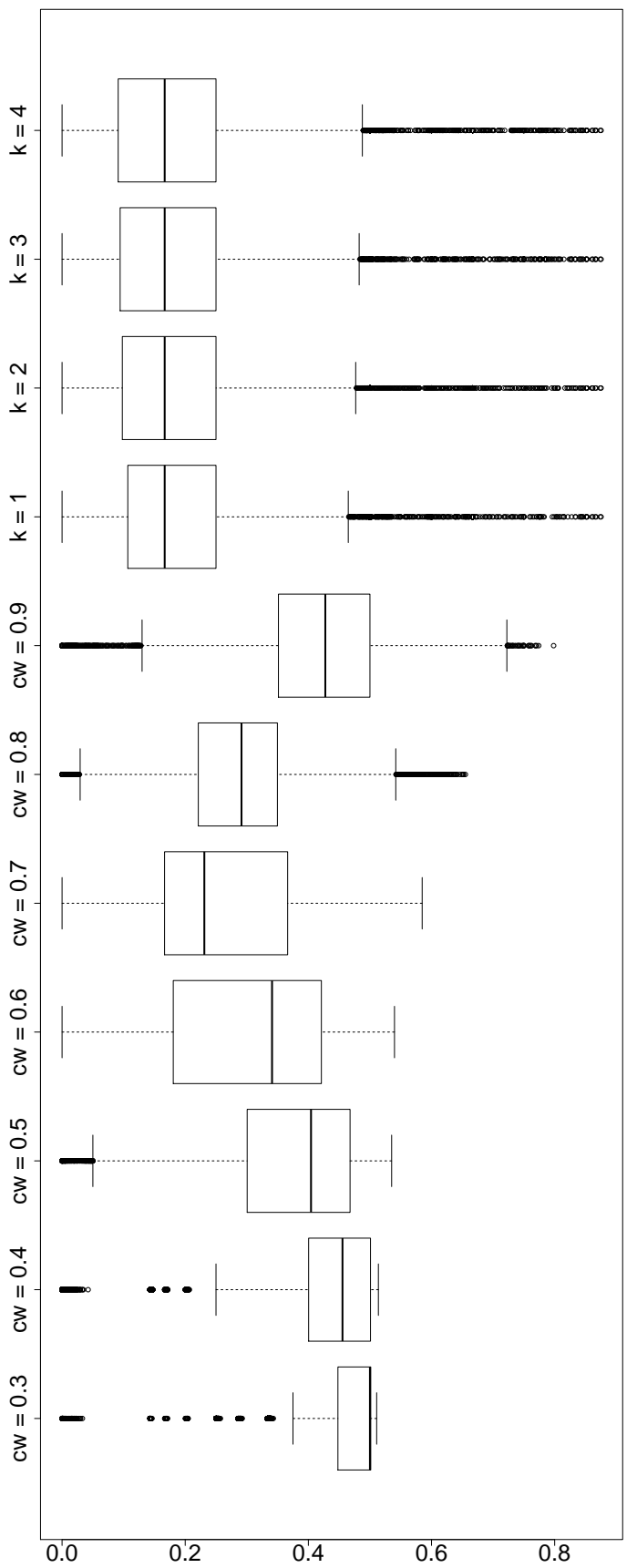

(b) EER

Figure 5.5: Effects of Classifier Parameters on Performance (AUC and EER) Shown with Boxplots (cw Refers to Fixed-Width Clustering Algorithm's Cluster Width Parameter, and $\mathrm{k}$ Refers to the $k$ in $\mathrm{k}-\mathrm{NN}$.) 


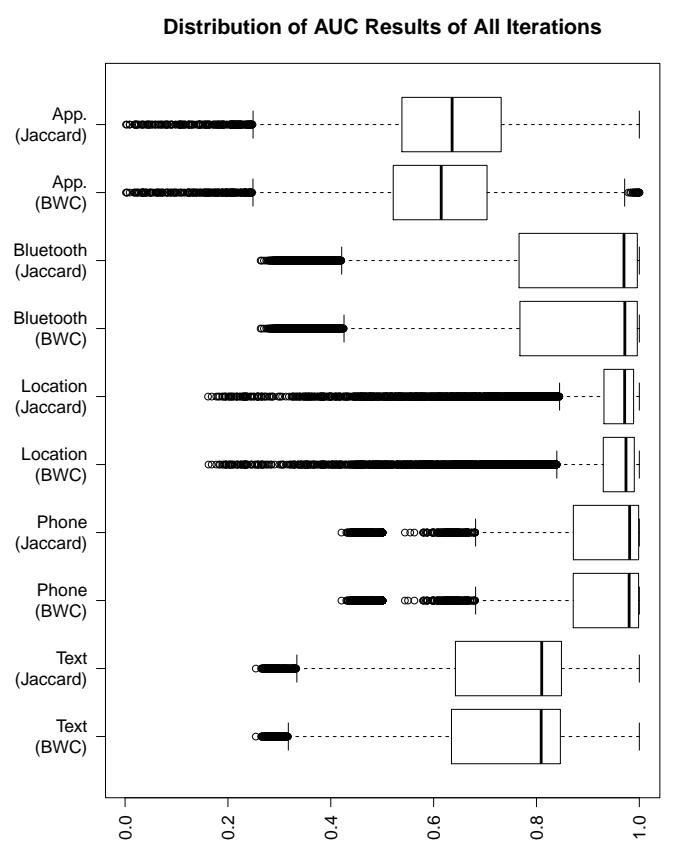

(a) Reality Mining Data Set - AUC

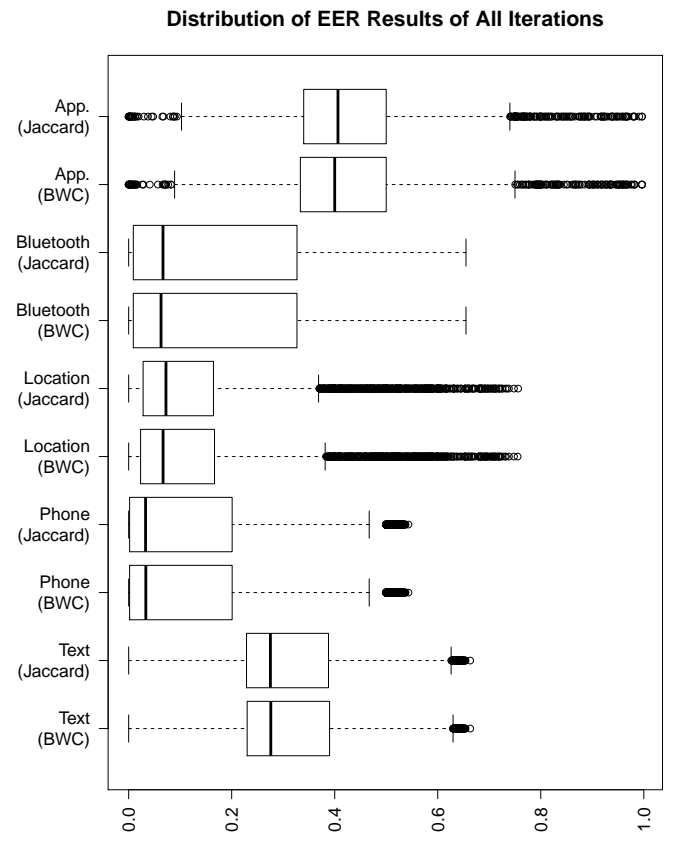

(b) Reality Mining Data Set - EER

Figure 5.6: k-NN Classification Performance (AUC and EER) of Different Features of the Reality Mining Data Set (k is 1, time slice length is 4 hours, "leniency" is 4 hours, training period length is 21 days) 


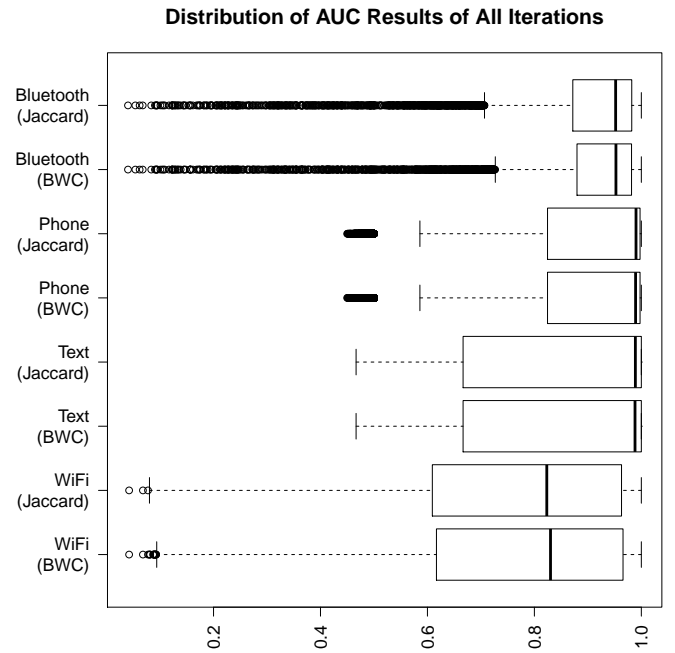

(a) Social Evolution Data Set - AUC

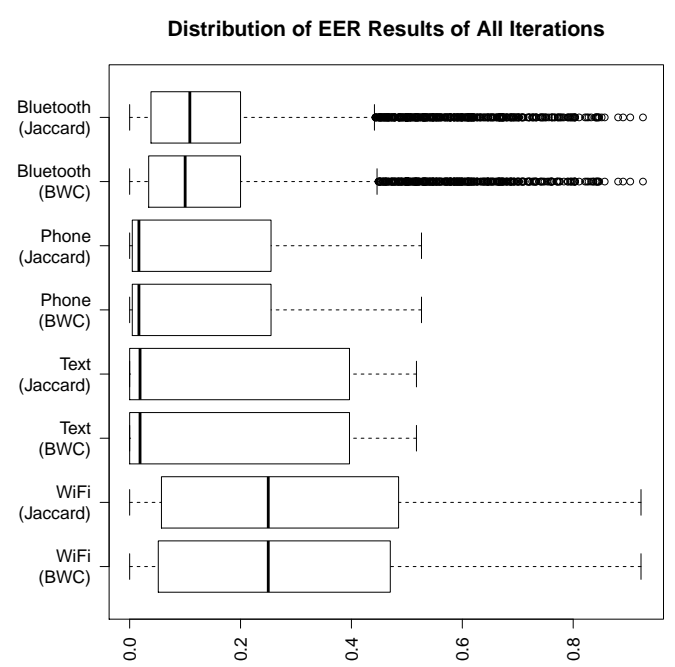

(b) Social Evolution Data Set - EER

Figure 5.7: k-NN Classification Performance (AUC and EER) of Different Features of the Social Evolution Data Set (k is 1, time slice length is 4 hours, "leniency" is 4 hours, training period length is 21 days)

Contributions of Individual Features As can be seen from Figure 5.6, based on the AUC and EER medians and the amount of spread from the medians, the phone call and location features appear to provide better predictive power in the Reality Mining data set, followed by the Bluetooth and text messaging features, and with the application feature providing possibly the least significant contribution to predictive power. For the location and phone call features, more than $75 \%$ of the iterations result in AUC values higher than 0.8, and for the Bluetooth feature more than $75 \%$ of the iterations result in AUC values higher than 0.75, as inferred from the top three quartiles in the boxplots.

According to the medians depicted in Figure 5.7, in the Social Evolution data set, the phone call and Bluetooth features perform better than text messaging and WiFi features. For the phone call and Bluetooth features, more than $75 \%$ of the iterations result in AUC values higher than 0.8, as inferred from the top three quartiles in the boxplots. 
For both data sets, we notice that the BWC metric does not appear to improve the results for most features, because of which we shall use the Jaccard distance metric for our remaining experiments.

To compare the performance between the two data sets, we see that the Social Evolution data set's Bluetooth feature spread is smaller than that of the Reality Mining data set, but with more outliers. For the phone call feature, we notice that the two data sets perform comparably with the Social Evolution data set having a larger spread. Finally, for the text messaging feature, we observe that the Social Evolution data set has a larger skew, but a higher median compared to the Reality Mining data set.

The reason for the relatively large spreads and outliers is the variation of the classification accuracy from test day to test day for each participant, and across different participants. As an example, to help explain the large spread of the Bluetooth feature in the Reality Mining data set, we present the AUC metric values of Reality Mining data set participants 7 and 19 in the form of scatter plots in Figure 5.8. As can be seen in Figure 5.8a, the application of our methodology on participant 7's data produces highly varying AUC metric results from test day to test day, whereas Figure $5.8 \mathrm{~b}$ shows that participant 19 can be authenticated based on the Bluetooth feature more reliably for the duration of the study.

Enabling All Features at Once As we will discuss later in Section 5.9, enabling all of the features at once does not, in our experiments, lead to a better performance than the best performing feature.

The rows in Figure 5.9 with the label "21 days" are the results of experiments with all of the features enabled and have the same configuration as the Reality Mining experiments in this section (Figure 5.6). As the reader will notice, while the overall performance is better than that of the individual low-performing features, the overall 

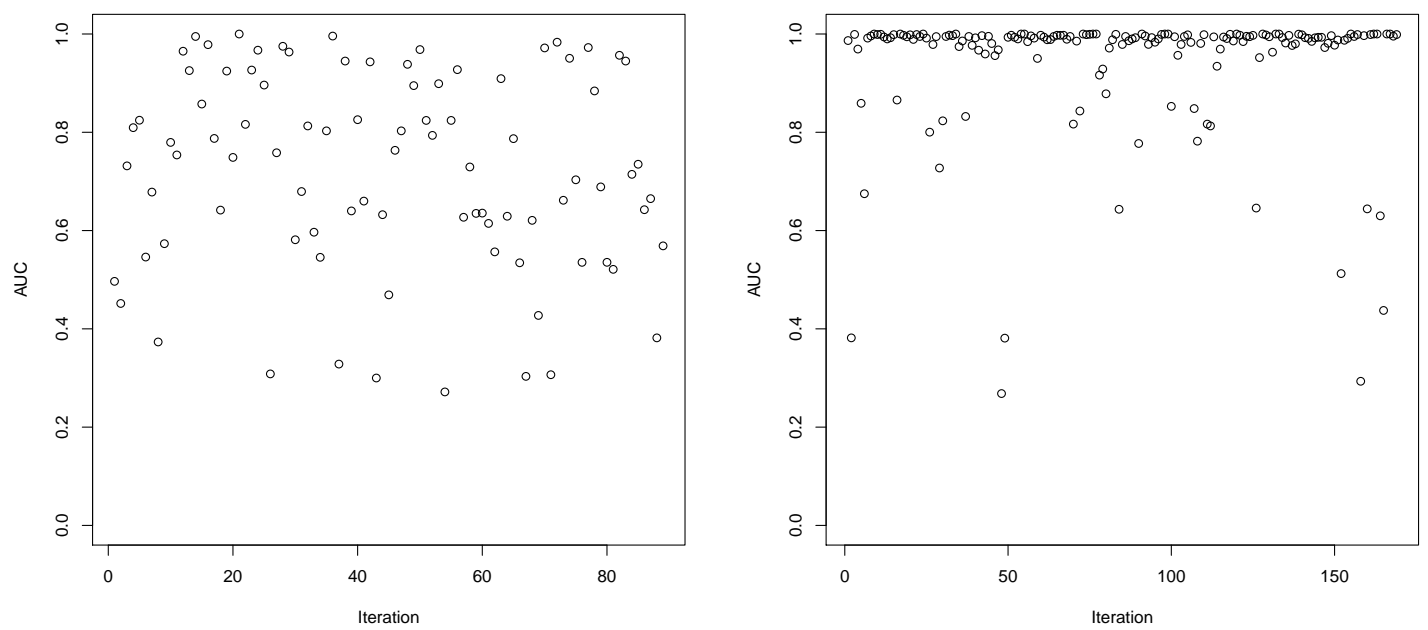

(a) Bluetooth feature for Reality Mining (b) Bluetooth feature for Reality Mining Participant 7 Participant 19

Figure 5.8: Scatter Plots of the AUC Metric Showing the Variance of the Metric between Iterations

performance does not appear to be better than that of the best performing features.

Similar conclusions can be made with regards to the experiments carried out with the Social Evolution data set in this section (Figure 5.7), and the same experiment with all of the features enabled, the results of which can be seen in the "21 days"labeled rows of Figure 5.10.

As we will explain in Section 5.9, this issue motivates us toward match score level fusion.

\subsection{Classification Performance with Different Training Period Lengths}

As was mentioned above, for each iteration or, in other words, each sliding-window shift of a sub-experiment, we train a one-class classifier on the $N$ days before "today." Here, we investigate whether training a classifier on only the past 1 day is sufficient, 
and whether varying the training period length from 1 day to 28 days makes a difference in the classification accuracy. For each data set and for each experiment, we use all features, and set $\mathrm{k}$ equal to 1 , the time slice length equal to 4 hours, and the distance function time slice "leniency" equal to 4 hours. We compute the distances between pairs of feature values using the Jaccard distance.

The results for the Reality Mining and the Social Evolution data sets are shown in Figures 5.9 and 5.10, respectively. Each experiment is composed of approximately 12000 iterations. We can see that for the Reality Mining data set, increasing the training period length from 5 to 28 days slightly decreases the AUC and the EER spreads, whereas the difference in the amount of spread between 1 day and 5 daylong training periods is visually more apparent. For the Social Evolution data set, we observe that changing the training period length has a stronger effect on the overall spread of the AUC and EER metric values.

Based on the results obtained with the Social Evolution data set in Figure 5.10, we will continue our experiments with 21-day-long training periods, where the duration of 21 days is chosen based on a trade-off between the time complexity of our experiments and the overall performance we obtain.

\subsection{Classification Performance with Different Time Slice Lengths}

To investigate the effect of different time slice lengths, we run a number of experiments, where we vary the time slice length from 0.5 hours to 4 hours. For each experiment, we use all of the features, set $\mathrm{k}$ equal to 1 , the distance function time slice "leniency" equal to 4 hours, and the training period length equal to 21 days.

To avoid very long experiment run-times, for these set of experiments, we use the 


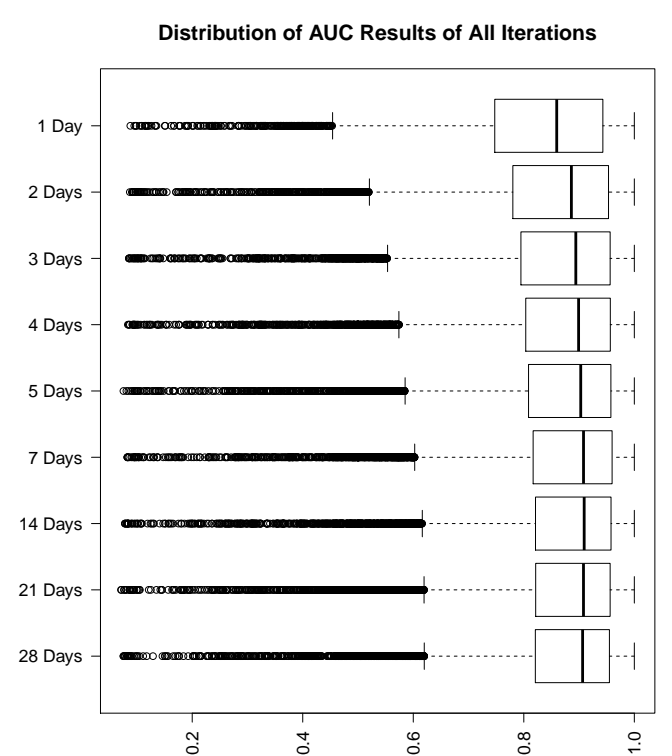

(a) Reality Mining Data Set - AUC

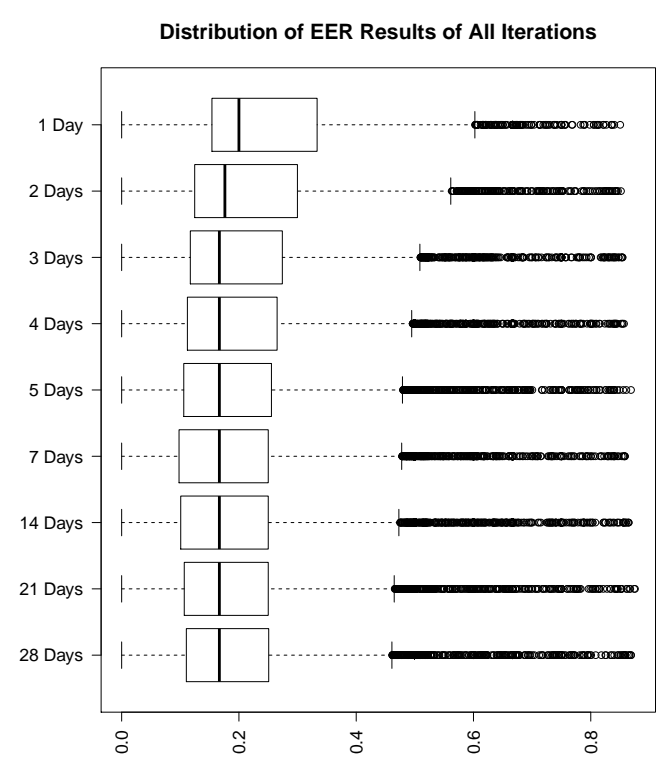

(b) Reality Mining Data Set - EER

Figure 5.9: k-NN Classification Performance (AUC and EER) of Different Training Period Lengths with the Reality Mining Data Set (All features, $\mathrm{k}$ is 1, time slice length is 4 hours, "leniency" is 4 hours)

"aggressive" sampling method which was explained in Section 5.4. The fact that we are using more aggressive sampling reduces the confidence with which we can draw conclusions from these experiments.

The results showing the classification accuracy with different time slice lengths are presented in Figure 5.11, by looking at which we notice that changing the time slice length appears to affect the classification performance, but we are not sure by how much, especially if one considers the relatively large spreads of the AUC and EER metric values.

To clarify the effect of the time slice length, we use two boxplots to compare the performance of 0.5-hour-long time slices to the performance of 4-hour-long time slices, where, for each paired iteration (test day), we subtract the AUC metric value resulting from the experiment with 4-hour-long time slices from that of the experiment with 0.5-hour-long time slices. This pairwise delta (or difference) boxplot lets us visually 


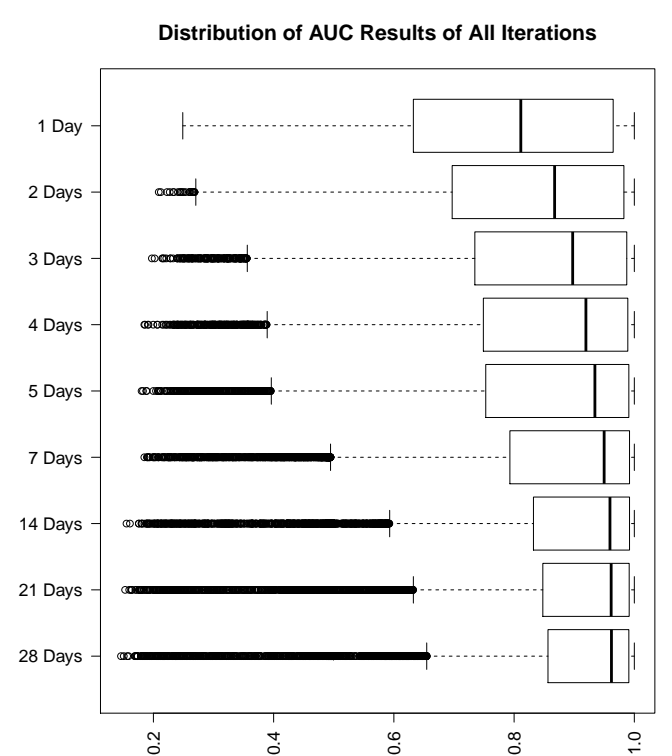

(a) Social Evolution Data Set - AUC

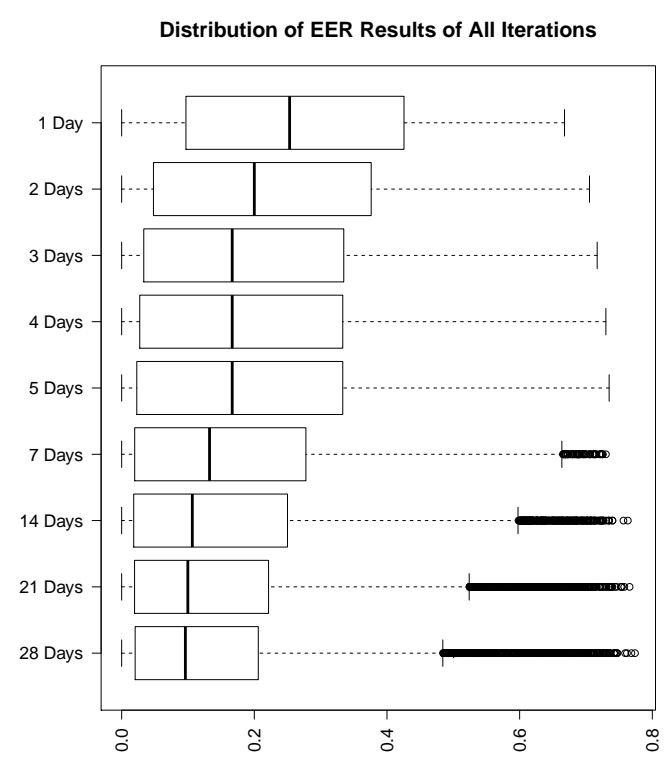

(b) Social Evolution Data Set - EER

Figure 5.10: k-NN Classification Performance (AUC and EER) of Different Training Period Lengths with the Social Evolution Data Set (All features, k is 1, time slice length is 4 hours, "leniency" is 4 hours)

see whether decreasing the time slice length creates a large difference in performance.

The plots are presented in Figure 5.12, via which we notice that there is not a clearly "winning" time slice length in this case. For both data sets, while the median delta is in favor of 4-hour-long time slices, the classification performance is sometimes better and sometimes worse as a result of reducing the time slice length. A majority of the test day results are within \pm 20 AUC percentage points of each other, which unfortunately is not conclusive.

\subsection{Results with Match Score Level Fusion}

As mentioned in Section 4.8, we experiment with different match score level fusion methods in an attempt to achieve improved results compared to our default method of feature level fusion. 


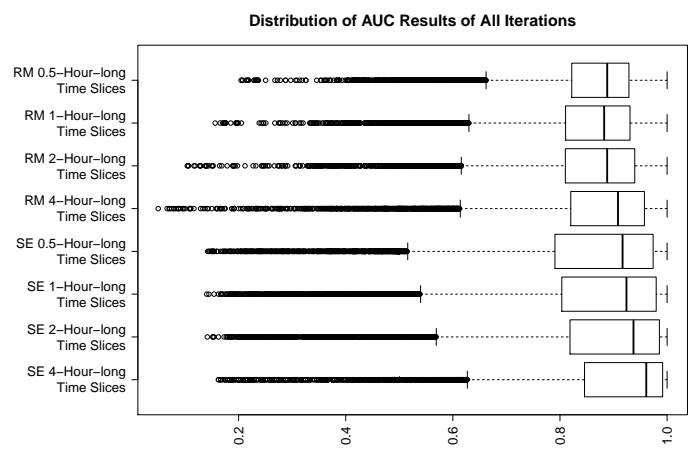

(a) AUC

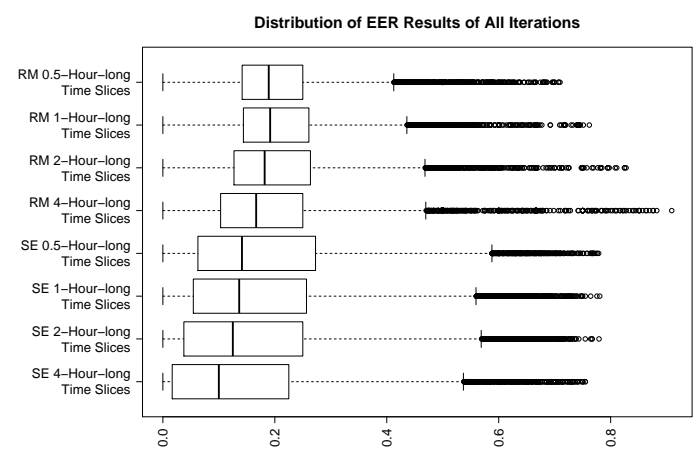

(b) EER

Figure 5.11: k-NN Classification Performance (AUC and EER) of Different Time Slice Lengths with the Reality Mining Data Set $(R M)$ and the Social Evolution Data Set $(S E)$ (Aggressive sampling, all features, $\mathrm{k}$ is 1, "leniency" is 4 hours, training period length is 21 days)

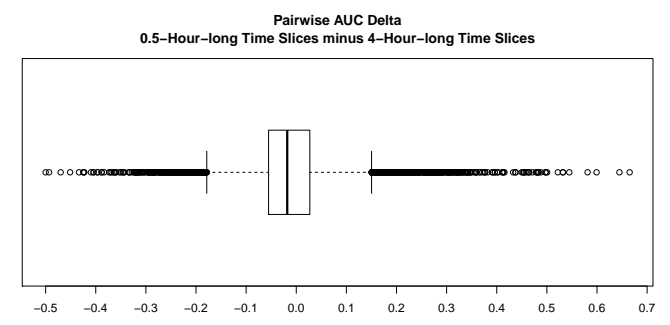

(a) Reality Mining (AUC)

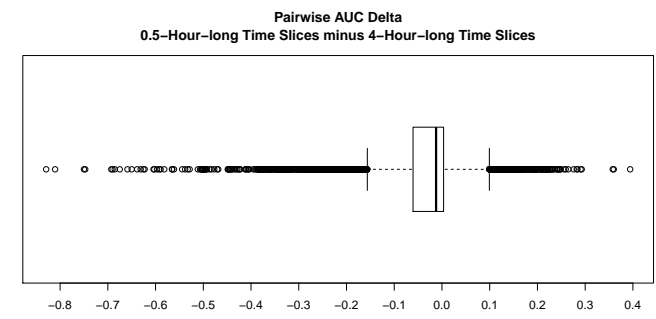

(b) Social Evolution (AUC)

Figure 5.12: Boxplots Depicting a Pairwise Comparison of the k-NN Classification Performance (AUC) per Iteration (or Test Day). (Aggressive sampling, all features, $\mathrm{k}$ is 1 , "leniency" is 4 hours, training period length is 21 days)

Our motivation comes from the fact that adding more features does not necessarily improve the accuracy of our method. For the Reality Mining data set, consider Figure 5.6, where we show the performance of our method with individual features. Now, consider the rows in Figure 5.9 which correspond to a 21-day-long training period length. As the reader will notice, with feature level fusion, our results tend to be closer to the average of the contributions that each individual feature provides, whereas we expect the overall performance with all of the features to be greater than "the sum of the individual parts." 
For these experiments, we use the k-NN algorithm with feature level fusion, and match score level fusion in the simple sum and voting configurations. We use all features, set $\mathrm{k}$ equal to 1 , the time slice length and the time slice length leniency equal to 4 hours, and use a training period length equal to 21 days.

We present the results from these experiments in Figure 5.13, where we notice that for the Social Evolution data set, the match score level fusion methods do not bring visually apparent improvements over the default feature level fusion method, whereas for the Reality Mining data set, the performance of match score level voting method appears to be slightly better than that of the feature level fusion method, when one considers the degree of spread for the AUC and EER metric values.

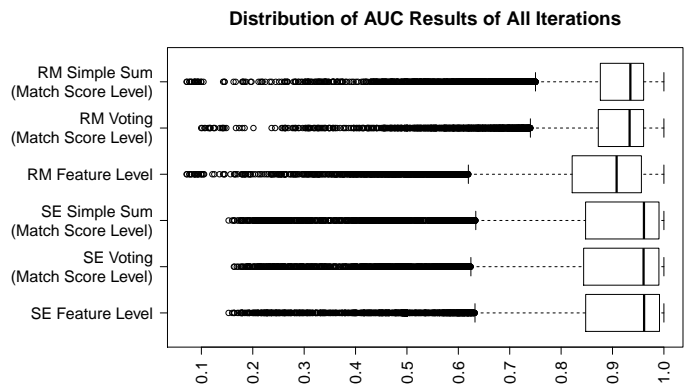

(a) AUC

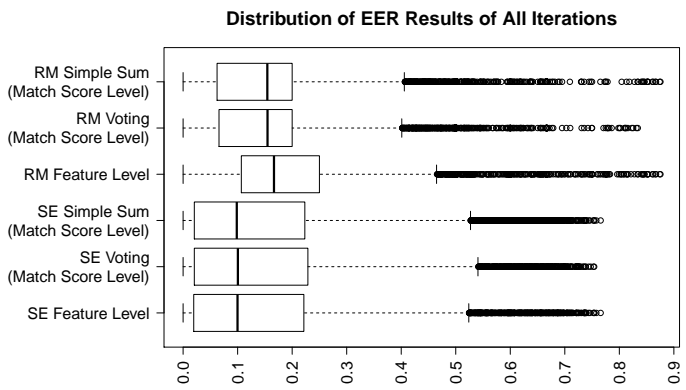

(b) EER

Figure 5.13: AUC and EER Results of Different Fusion Methods with k-NN and the Reality Mining Data Set $(R M)$ and the Social Evolution Data Set $(S E)$ (All features, $\mathrm{k}$ is 1 , time slice length is 4 hours, "leniency" is 4 hours, training period length is 21 days)

To investigate the validity of the visual intuition discussed in the previous paragraph, we use a pairwise delta (or difference) boxplot between the AUC results of match score level voting and feature level fusion methods. In Figure 5.14, we notice that while the median of the pairwise differences is greater than zero, indicating that match score level voting has some advantage, the overall spread is between \pm 10 AUC percentage points, preventing us from drawing conclusions regarding the superiority of match score level fusion in this case. 


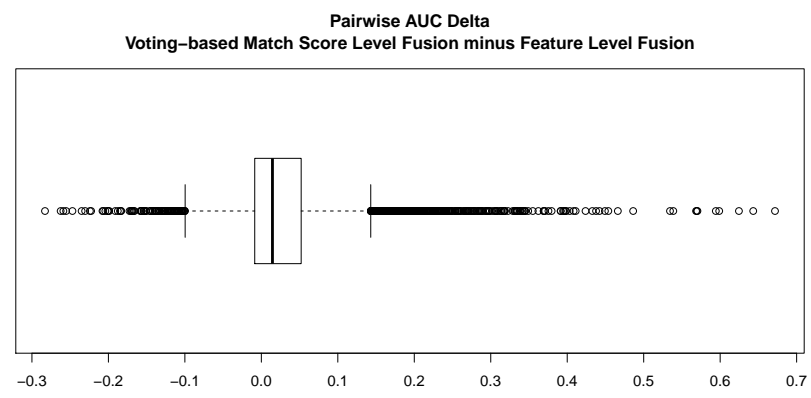

Figure 5.14: Boxplot Depicting a Pairwise Comparison of Classification Performance (AUC) per Iteration (or Test Day) of Match Score Level Voting and Feature Level Fusion with the Reality Mining Data Set. (All features, $\mathrm{k}$ is 1 , time slice length is 4 hours, training period length is 21 days)

\subsection{Comparison to a Method without Time Slices}

Our method is heavily reliant on the time slice notion to summarize the observations of a mobile device in terms of the behavioural patterns of its owner. As discussed in the Related Work chapter, in [10] and [11] Li uses one of the data sets that we use for evaluation (the Reality Mining data set), and carries out anomaly detection with a relatively simple rule-based approach which does not take into account the time of day at all, and only considers the prevalence of each feature value in the training data, while also fusing the location feature with the application, phone call and text messaging features.

To compare these two approaches, we implement a rule-based method similar to the one used by Li, with the exception that our implementation does not have a "smoothing function" which groups together a configurable number of "contextual events."

\subsubsection{Overview of the Method without Time Slices}

The rule-based method we implement operates in either "location feature fusion mode," or in "plain mode." 
- In location feature fusion mode, for each application, Bluetooth, phone call and text messaging feature value feature $_{i x}$ that occurs during the training period, the implementation fuses the most recent unexpired location feature

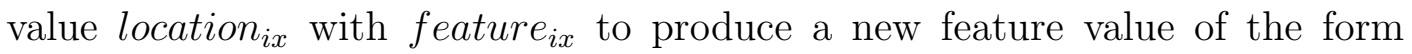
$\left(\right.$ feature $_{i x}$, location $\left._{\text {ix }}\right)$.

- In plain mode, no feature level fusion is performed, and instead the location feature is considered a regular feature.

Afterwards, we build one hash table per feature type, each of which contains the prevalence ratios of each of the aforementioned feature values. Each hash table is keyed with the feature values seen in the training period. Each key in a hash table points to a value representing the ratio of the number of occurrences of the feature value in question over the number of occurrences of all of the feature values for that feature type in the training period. In summary, the mentioned hash tables summarize how common the feature values seen in the training period were.

In the testing phase, if a feature value which does not exist in the appropriate hash table is encountered in a test day (which we also call an iteration), then the feature value is predicted to be an attack "event" observed by the mobile device. If the feature value is found in its feature type's hash table, then we assign this "event" a score equal to its prevalence ratio.

Finally, we plot ROC curves and obtain the AUC and EER metric values by varying a threshold score and comparing the labels of the feature value events to the predicted labels, where if an event has a score below the threshold, then it is predicted to be an attack; otherwise, it is predicted to be normal. 


\subsubsection{Evaluation Overview}

For evaluation purposes, we only use the Reality Mining data set, as the Social Evolution data set does not have a location feature in the form of cellular tower identifiers.

We use the evaluation method explained Section 5.1 to compare our time slicebased method with the rule-based method, with the exception that for the rule-based method we sample feature value events from the pool of all feature value events belonging to all attackers. We feel that such an approach is necessary as the rulebased method does not take into account the time of day and does not have the "notion" of a time slice.

Based on the observation that there does not exist a day with more than 2000 feature value events for any user in the Reality Mining data set, for each iteration, we arbitrarily sample 20,000 positive feature value events from the pool of data of all attackers with the rule-based method. For both methods, we use all features and a training period length of 21 days. For the time slice-based method, we set k equal to 1 , select 1-hour-long time slices with a leniency of 4 hours, and we use aggressive multi-stage cluster sampling.

\subsubsection{Results}

As can be seen in Figure 5.15, in terms of the AUC metric, the median and the third quartile of the time slice-based method are higher than those of the rule-based methods (with and without location feature fusion). However, we also notice that the time slice-based method does not appear to be better than the rule-based method with location feature fusion - the time slice-based method has a narrower spread, but also more outliers.

We compare the performance of the time slice-based method to the rule-based 


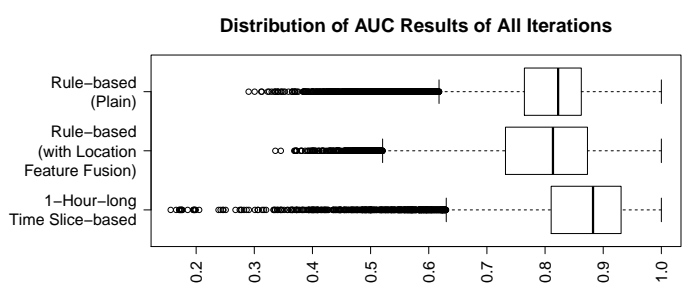

(a) AUC

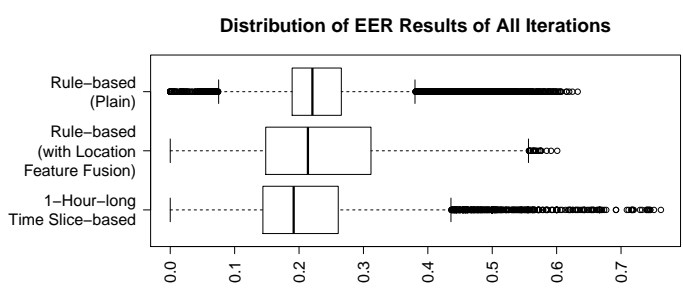

(b) EER

Figure 5.15: AUC and EER Results of the Two Rule-based Approaches and the 1-Hour-long Time Slice-based Approach with the Reality Mining Data Set.

method with location feature fusion using a pairwise delta (or difference) boxplot on the AUC and EER metric values in Figure 5.16.

With the AUC metric, it appears that a larger portion (the second and the third quartiles and the lower whisker) of the pairwise differences favor the 1-hour-long time slice-based approach, even though for some iterations, it appears that the rule-based method with location feature fusion performs better.

In contrast, with the EER metric, even though a portion of the pairwise differences are positive - indicating that the rule-based approach has higher EER and lower accuracy - we also see that the median of the pairwise differences is closer to zero than that of the AUC metric values.

As a result, based on the two sets of results, we are unable to confidently state whether the time slice-based method performs more accurately than the rule-based method with location feature fusion.

\subsubsection{Limitations}

There are two limitations of the experiments comparing the rule-based approaches to our time slice-based approach:

1. The sampling rate that we use with the rule-based approaches to sample the positive (attacker) feature value events is arbitrarily chosen, and has not been 


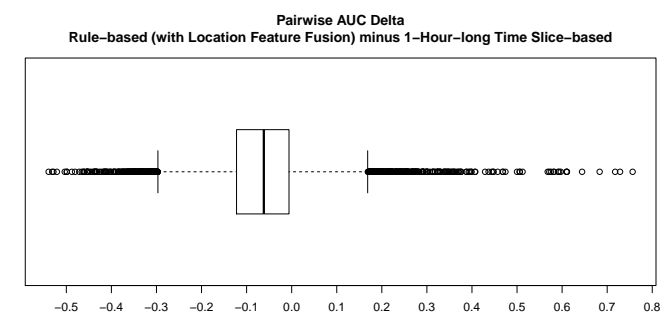

(a) AUC

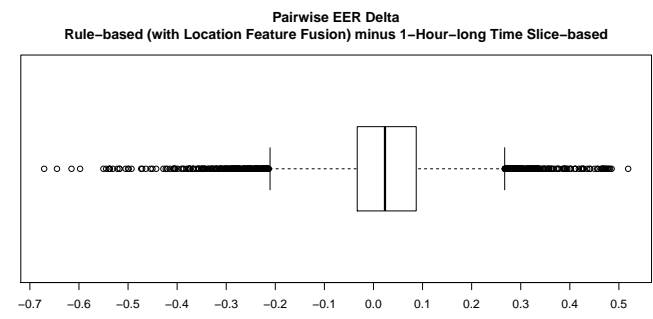

(b) EER

Figure 5.16: Boxplots Depicting a Pairwise Comparison of Classification Performance (AUC and EER) per Iteration of 1-Hour-long Time Slice-based Method and Rulebased Method with Location Feature Fusion (on the Reality Mining Data Set).

validated. As a result, it is not possible to conclude with a high level of confidence whether the two methods are equivalent or not.

2. More importantly, unlike Li's approach in [11], in our implementation of the rule-based method, we did not use a "smoothing function" to group together feature value events in order to increase the accuracy of the said method.

Given that Li observes a significant decrease in the EER of the rule-based method when 6 events are grouped together compared to an approach with no grouping of events, our results may not be representative of the best possible performance of the rule-based method.

\subsection{Comparison of Our Results with Those of Li}

Because of the aforementioned limitations of our implementation and evaluation of the rule-based methods, we also compare our results directly with the results obtained by $\mathrm{Li}$ in his $\mathrm{PhD}$ thesis [11].

We notice that the experimental set-up was not explained in great detail in $[11$, p. 79]. For example, we do not know whether the reported EER values are the means of the EER values resulting from the performed experiments, and how the positive (that is, attacker) feature value events were selected out of the pool of all attackers' 
data.

Li does note in $[11$, p. 77$]$ that the rule-based approach is evaluated only on the portion of the Reality Mining data set which corresponds to the most dense part of the data set in terms of the number of participants for whom data exists: the period between 24th of October, 2004 and 20th of November, 2004.

Li experiments with the application, phone call and text messaging features individually fused with the location feature, after which all of the features are combined in a final experiment documented in [11, p. 110], which is the experiment that we will base our comparison on. For this experiment, Li notes that there was data from 76 participants available. "Static profiles" are experimented with as well, but for comparison reasons, we focus on the "dynamic profiles."

In contrast, in our approach we fuse the application, Bluetooth, location, phone call and text messaging features at the feature level (or match score level), and we utilize 95 participants' data from the complete data set.

The results reported by $\mathrm{Li}$ for this experiment are reproduced without modification in Table $5.1^{2}$, where the "Number of Log Entries" corresponds to the number of events that were grouped together to increase the accuracy, and the number of days mentioned in the "Profile Technique" column refer to the length of the training period.

\begin{tabular}{|l|l|l|l|l|l|l|l|}
\cline { 3 - 8 } \multicolumn{2}{c|}{} & \multicolumn{7}{c|}{ Number of Log Entries } \\
\cline { 3 - 8 } & 1 & 2 & 3 & 4 & 5 & 6 \\
\hline \multirow{3}{*}{ Profile Technique } & Static 14 days & $16.9 \%$ & $13.6 \%$ & $12.7 \%$ & $12 \%$ & $10.9 \%$ & $11 \%$ \\
& Dynamic 7 days & $19 \%$ & $15.2 \%$ & $13.1 \%$ & $12.4 \%$ & $11.3 \%$ & $10.5 \%$ \\
& Dynamic 10 days & $17.4 \%$ & $13.7 \%$ & $12.3 \%$ & $11.6 \%$ & $10.6 \%$ & $9.8 \%$ \\
& Dynamic 14 days & $16.5 \%$ & $13.5 \%$ & $12.1 \%$ & $11.6 \%$ & $10.5 \%$ & $10.1 \%$ \\
\hline
\end{tabular}

Table 5.1: Li's Results in Terms of the EER Metric ${ }^{2}$

According to Table 5.1, the lowest (and therefore the best) EER metric value of

\footnotetext{
${ }^{2}$ As appears on [11, p. 110].
} 
9.8\% was obtained when a dynamic user profiling method with a 14-day-long training period was utilized in conjunction with the grouping of 6 events.

In contrast, our best mean and median EER metric values with feature level fusion are obtained with a training period length of 28 days and 4-hour-long time slices, as can be seen in Figure 5.9. The median EER metric value in the mentioned experiment is approximately $16.7 \%$, whereas the mean is approximately $19.5 \%$.

Our best EER mean and median metric values with match score level fusion are obtained with the simple sum fusion method, 4-hour-long time slices, and a training period length of 21 days, where the mean EER is $15.7 \%$, and the median EER is 15.4\%. These results can be seen in Figure 5.13.

Discussion Now that we have reported our results and Li's results, we would like to note that the comparison of the performance of the two methodologies using only the means (or the medians) is far from ideal, as there can be significant differences from test day to test day and between different data set participants, which is depicted in most of the boxplots in this document. Furthermore, the differences in the ways that $\mathrm{Li}$ and we use the Reality Mining data set are also significant. As a result, the conclusions that can be drawn from the comparison of single-number summaries are very limited.

With these in mind, Li's method appears to be more accurate in terms of the reported raw EER values.

\subsection{Conclusion}

In this chapter, we have evaluated our methodology with the Reality Mining and the Social Evolution data sets using dynamic user profiles and with feature level fusion and match score level fusion. 
We see that the sampling method used to reduce the time complexity of the evaluation only slightly affects the evaluation results, which indicate that k-Nearest Neighbours performs better than the fixed-width clustering algorithm.

We also find that the predictive power of the location (in the form of cellular tower identifiers) phone call, and Bluetooth features are higher compared to the other features in the Reality Mining data set, and that the predictive power of the phone call and Bluetooth features are higher compared to those of the text messaging and WiFi features in the Social Evolution data set.

Furthermore, we cannot conclude positively or negatively on whether the the time slice length (when varied between 0.5 and 4 hours), affects the classification results significantly. The training period length - when varied between 1 and 28 days affects the results, with longer training period lengths performing slightly better.

In an attempt to increase the robustness of our approach, we run experiments comparing our default fusion method of feature level fusion with match score level fusion, according to the results of which we cannot conclude that match score level fusion significantly improves the performance, especially with the Reality Mining data set.

As noted before, our method heavily relies on the notion of time slice-based summarization. We compare our approach to one which does not use time slices. It is found that the performance of the two methods cannot reliably be distinguished. We also compare our results with Li's results, finding (with some reservations) that based on the raw overall EER metric values reported by Li, Li's method might be more accurate than ours.

Finally, as noted in the chapter, the performance of our method may vary significantly from user to user and from test day to test day. This indicates that further work is needed in order to increase the robustness of our method. 


\section{Chapter 6}

\section{Deployment}

In this chapter, we discuss our experience with the implementation, deployment and evaluation of our method on an actual mobile device. We first discuss the chosen target mobile device platform, which is followed by an overview of the experimental set-up used to evaluate the deployed version of our software. Next, we discuss the results, and finally, we conclude the chapter.

\subsection{Target Mobile Device Platform}

While preparing to deploy the software implementing our method on a mobile device, we had in mind two target platforms: (1) Nokia's Linux-based Maemo operating system, which runs on the Nokia N900 - a discontinued smartphone introduced in 2009 - and (2) Google's Linux-based Android operating system, which runs on a large variety of devices available as of this writing.

We evaluated the mentioned platforms according to the following criteria: (1) Ease of development, especially with respect to the amount work that would be needed to port our Python-based software to the platform in question, (2) our familiarity with the platform, and lastly, (3) the suitability of the chosen platform and the mobile device for our purposes in terms of software and hardware. 
At the time of the development, we had extensive experience with Python and Maemo, whereas our experience with Java (the primary programming language used to develop for Android) and the authoring of Android applications was not extensive enough to provide sufficient confidence. ${ }^{1}$ Based on our experience, we were aware of the possibility that we could simply copy our code to the N900, whose default Python 2.5 installation would be able to run the code with almost no modification.

In terms of the first two criteria, the Maemo-based Nokia N900 is very suitable for our purposes; however, for the third criterion, we should acknowledge that Android can be more suitable, given that Android makes it very simple to access sensors for the acquisition of data, and more importantly, as a non-obsolete platform, there are a large number of Android-based mobile devices available as of the time of this writing, whereas Nokia's discontinued N900 is no longer available.

Finally, given that the N900 is a smartphone from the year 2009, its hardware capabilities are significantly limited compared to more modern smartphones. We summarize the hardware specifications relating to the computation and storage capabilities of the N900, and LG Nexus 4 - a relatively recent and high-end Android-based smartphone as of this writing - in Table 6.1.

\begin{tabular}{|l|r|r|}
\cline { 2 - 3 } \multicolumn{1}{c|}{} & Nokia N900 & LG Nexus 4 \\
\hline Number of CPU Cores & 1 (One) & 4 (Four) \\
\hline CPU Clock Frequency & $600 \mathrm{MHz}$ & $1.5 \mathrm{GHz}$ \\
\hline $\begin{array}{l}\text { Random Access Memory } \\
\text { (RAM) Capacity }\end{array}$ & 256 MegaBytes & 2 GigaBytes \\
\hline $\begin{array}{l}\text { Persistent (Flash) Mem- } \\
\text { ory Capacity }\end{array}$ & 32 GigaBytes & 8 or 16 GigaBytes \\
\hline
\end{tabular}

Table 6.1: Comparison of the Capabilities of Our Target Smartphone to Those of a Recent Smartphone

\footnotetext{
${ }^{1}$ We should note that it is possible to run Python on Android via the Scripting Layer for Android (SL4A) framework which is available at: https://code.google.com/p/android-scripting/
} 
As can be seen in Table 6.1, in terms of all mentioned hardware except the flash memory capacity, LG Nexus 4 is more capable than Nokia N900 by at least one order of magnitude.

While the obsolescence of the N900 limits what we can achieve with deployment, we would like to note that if our software runs acceptably on the N900 with Python, which is an interpreted language, then we can (with a high degree of confidence) predict that the overall performance of our method will be much more successful with a modern smartphone.

\subsection{Experimental Set-up}

In this section, we outline our experimental set-up, including a description of the features collected by the deployed version of our software, the data collection parameters and anomaly detection method, and finally an explanation of the experiments we performed.

\subsubsection{Collected Features}

Currently, our software collects and performs anomaly detection on only Bluetooth and WiFi discovery results. From the discovery results, we select the following pieces of information to identify Bluetooth and WiFi devices: (1) For Bluetooth discovery: the the human-readable name and the Bluetooth (MAC) address of each discovered device, and (2) for WiFi discovery: the ESSID (i.e. the human-readable name broadcasted by the access point), BSSID (i.e. the MAC address of the access point), and the type of encryption (i.e. "open," "WEP," "WPA1," and "WPA2") of each discovered wireless network.

As the reader will recall from the Methodology chapter, the feature values sensed by the individual sensors (Bluetooth and $\mathrm{WiFi}$ ), are represented as tuples of the 
above-mentioned pieces of information. For example, if during a particular time slice, the N900 discovers two WiFi access points and one Bluetooth device, then the current time slice could include the feature value tuples of the following form:

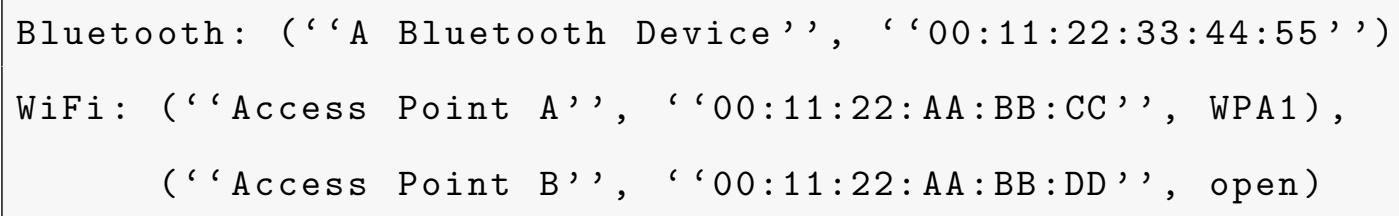

\subsubsection{Data Collection and Anomaly Detection}

We collect the above-mentioned feature values at different rates, which are independent from the length of a time slice. When a time slice ends, we provide this time slice to the anomaly detection algorithm so that it can be classified as normal or anomalous.

In our experiment, Bluetooth discoveries are performed every 25 seconds, whereas WiFi discoveries are performed every 30 seconds. We set the time slice length to 1 minute. We consider a training period length of 5 days, meaning that only time slices that occurred in the past 5 days of "today" are provided as training data to the anomaly detection algorithm.

As the reader will notice, the above mentioned data collection parameters and anomaly detection method involve a slight departure from those used in the Evaluation chapter. Given the limited time we had for deployment, we choose relatively small lengths of time for the discovery periods and the time slice length in order to have a "worst case" look at the resource usage, in terms of CPU and memory utilization and battery life.

In addition, we opt to use an unoptimized variant of Eskin et al.'s k-NN algorithm with $k$ set equal to 1 , and at the end of each time slice, we classify the time slice which has just ended, after which we "retrain" the anomaly detection algorithm to include 
the time slice that was classified. ${ }^{2}$ We should note that the fixed-width clustering algorithm, while not evaluated, is deployed as well.

After each anomaly detection run, our software reports in text form on a terminal the "anomaly score" of the time slice which had just been classified.

\subsubsection{Description of Experiment}

We ran an experiment for approximately one week, during which we used an N900 frequently as a personal music player, and to make occasional phone calls, while our deployed software ran in the background for the duration of the experiment.

During this experiment, we qualitatively evaluated the user experience, and CPU and memory utilization were observed using a real-time "applet" software which was placed on the desktop of the smartphone's graphical user interface. The consumption of battery was recorded using a third-party battery level logging software, named battery-eye.

After the experiment, we quantitatively evaluated the resource usage of our software in terms of CPU utilization, random access memory utilization, persistent (flash) memory usage, and battery consumption.

\subsection{Results and Discussion}

From the subjective perspective of a user for the duration of the experiment, in terms of the computational performance of the N900, we could not notice that our software had been running in the background. For example, we never experienced interruptions because of an anomaly detection run during a phone call or while playing music, even

\footnotetext{
${ }^{2}$ We quote the word "retrain" in this sentence, because with an unoptimized k-NN algorithm, the training process only consists of storing the training data, which demands a relatively small amount of CPU usage.
} 
though we could clearly observe the CPU usage rise when our software was classifying time slices.

The battery life was far from ideal, however. Based on the logs of the battery level logging software, we needed to recharge the battery approximately every 15 or 16 hours, whereas the N900's specifications indicate that its stand-by time can ideally be 10 days. Nevertheless, the issues with the battery life have been expected given the relatively frequent Bluetooth and WiFi scans, and given that an anomaly detection operation was performed every minute.

In terms of CPU utilization, with a training set composed of one-minute-long time slices belonging to 5 days, the classification of one time slice takes 6 to 7 seconds, indicating that it may have been a better decision to use an incremental version of the fixed-width clustering algorithm or the optimized k-NN algorithm introduced by Eskin et al. in [3], in conjunction with an increase in the length of the time slices.

In terms of random access memory utilization, we observe that with the same data set loaded in memory, our software's Resident Set Size (RSS), as reported by the Linux kernel, is approximately 65 MegaBytes, which we believe is acceptable given the significantly larger amount of memory available on modern smartphones.

Finally, in terms of the consumed persistent (flash) memory, the SQLite databases containing about 9 days' worth of Bluetooth and WiFi discovery data consume approximately 6 and 26.5 MegaBytes, respectively. Even though with each additional feature we would have a larger amount of persistent memory consumption, we believe that with the much larger capacities of modern mid- to high-end smartphones, often measured in GigaBytes, the amount of data we need to store for anomaly detection purposes is acceptable. 


\subsection{Conclusion}

In this chapter, we have discussed the selection of a target platform on which to deploy our anomaly detection implementation, after which we have covered the experimental set-up, and finally, the results of our experiment.

We chose to use Nokia N900 as the target platform for deployment despite its obsolescence in terms of hardware because of our extensive development experience with it using Python. After deploying our software, we ran a modest one-week-long experiment where anomaly detection was performed based on Bluetooth and WiFi discovery results. The experiment was configured with fairly aggressive CPU and battery utilization requirements, on purpose.

The experiment results indicate that using our implementation does not, in qualitative terms, negatively impact the user experience in terms of CPU and random access memory utilization.

In terms of battery consumption, we note that there is room for improvement, possibly via the use of an optimized variant of the k-NN algorithm, or the fixedwidth clustering algorithm, and possibly by increasing the time slice length, which had been set equal to one minute.

Finally, in quantitative terms, we observe that the overall random access memory usage and persistent (flash) memory requirements are acceptable.

In summary, we believe we have shown in this chapter the feasibility of the deployment of our anomaly detection method on an actual mobile device. While our results may be device-specific, we believe it is possible to obtain similar, if not better, results with recent mid- and high-end smartphones. 


\section{Chapter 7}

\section{Conclusion}

In this thesis, we have addressed the research problem "How can a comfort-enabled mobile device detect anomalies in the context, and the behavioural patterns of its user?" by making the following contributions, each addressing one of the research sub-problems we have identified in Chapter 1:

1. We have summarized contextual and behavioural patterns of a mobile device user using our time slice model, and combined the time slice model with two of Eskin et al.'s [3] anomaly detection algorithms and a custom distance function specific to the time slice model. We have evaluated the resulting method on the Reality Mining and Social Evolution data sets.

2. By evaluating the time slice model on the Reality Mining and Social Evolution data sets, we have observed that the location (in the form of cellular tower identifiers), phone call history, and Bluetooth discovery features perform with better accuracy when used with the described method, in comparison to the application usage, text message exchange history, and WiFi discovery features.

3. Finally, we have confirmed the applicability of our method on a mobile device with modest hardware specifications by deploying our software on a Nokia N900 smartphone and carrying out an experiment with qualitative and quantitative results. 
Given the positive results we have obtained from our evaluations, we believe that we have sufficiently answered our research question, the contributions resulting from which are useful and highly applicable to the Device Comfort architecture and validate the architecture's effectiveness when used with different devices in different contexts.

We have identified a number of future work items based on this research, which are addressed in the following section.

\subsection{Limitations and Future Work}

We can identify the following limitations of our work, leading to a number of future work items:

- The Reality Mining data set was collected during the 2004-2005 academic year, whereas the Social Evolution data set was collected during the 2008-2009 academic year. Furthermore, both data sets contain data depicting the mobile device usage patterns of students, faculty and staff of the Massachusetts Institute of Technology in the United States, which is where both data sets were collected.

The implications of the above-mentioned are that the data contained in these data sets may not represent the recent mobile device usage patterns of the general population, which we believe is evolving over time, and may show different characteristics in different locales.

As a result, the conclusions we can draw from the evaluation of our anomaly detection method with the two data sets are limited, and for these reasons, we believe that the collection and publishing of more recent data sets from a variety of social and cultural settings would be an important general contribution to this field.

- As part of future work, we believe that our method can be modified to enable 
support for time slices which vary in size. For example, when the mobile device user is sleeping at night, we might opt to use a longer time slice to summarize that part of the day.

Related to this modification is the detection of anomalous events as they occur, where, instead of waiting for a time slice to end in order to report an anomaly, our method could be extended to classify (at the cost increased computational complexity and hence energy consumption) the events as they occur by comparing the events to those in the time slices in the training data.

- The metrics used to evaluate our method - namely, AUC and EER - involve assigning equal weight to false positives (i.e., normal behaviour detected as an attack) and false negatives (i.e., anomalous behaviour detected as normal); however, depending on the field of application of our method, such an assumption may not hold true.

For example, in an application for very secure settings, false negatives can be very costly. In contrast, in a personal use setting, large number of false positives may result in the frustration of the user.

The evaluation of our method with metrics that assign different weights to false positives and false negatives is another future work item, which can be conducted based on the target application setting.

- As mentioned in Chapter 5, and noted in its conclusion, the results of evaluating our method on the two data sets indicate that there is a relatively high degree of variability in the overall accuracy of our method, in day-to-day device usage patterns of individual data set participants, and across different participants. This issue may need to be resolved via the refining of the time slice model, for example through the use of different distance functions. One direction for future work could be the investigation of weighting individual features according to their contribution to the overall accuracy, which could be customized per user. 
- As discussed in Chapter 5 to motivate match score-level fusion, the performance of our method with all features enabled is not better than the performance of our method with only the best-performing feature. This can either be because of our method or because of the data sets.

To address the former, one future work item is the experimentation with different methods for combining features in order to preserve accuracy as more features are used.

For the latter, we envision the verification of our method via synthesized user studies, in which one group of users could be instructed to use their mobile devices in a manner which produces sensor data from multiple sensors within the same time slice, and another group of users could be instructed to use, for example, only the phone feature of their mobile devices.

- The evaluation of our method (Chapter 5) was conducted in a "greedy" manner, where we first experimented with one parameter, after which we used the parameter value which produced the highest accuracy or the lowest error rate for the remaining experiments. The order of the experiments we conduct in this greedy method may result in a local optimum in terms of the results we obtain. For this reason, another future work item is the verification of our results by conducting the parameter selection experiments in one or more different orders.

- As discussed in Chapter 6, we have only experimented with one-minute-long time slices when evaluating the deployment of our method on a Nokia N900, the main aim of which was to confirm the feasibility of deploying our method, but not its accuracy in a deployed setting.

Future work can involve experimenting with different time slice lengths while conducting experiments on actual hardware to confirm the usability of our method in terms of its accuracy. 
- Another future work item is the deployment of our approach on modern hardware to confirm our conjecture that with such hardware it would be possible to obtain the same or better performance with regards to anomaly detection run-time length and battery consumption, possibly with longer time slices.

- Last, but not least, the integration of our method to an implementation of the Device Comfort architecture is an important future work item as well.

\subsection{Conclusion}

To conclude, Device Comfort is a paradigm which aims to protect the information on mobile devices, the mobile devices themselves, and potentially their users by bringing together the user-device relationship with the concepts of trust, context and reasoning.

A large amount of data and highly capable reasoning is needed by a mobile device to detect its user(s), verify (or recognize) their identities, and to predict the context. The work in this thesis is one step toward fulfilling these needs by enabling mobile devices to reason with sensor data which they are more than capable of collecting and processing.

In the future, we hope to see this work integrated with the Device Comfort architecture and tested and refined for modern mobile devices. 


\section{List of References}

[1] S. Marsh, P. Briggs, K. El-Khatib, B. Esfandiari, and J. Stewart, "Defining and Investigating Device Comfort," Journal of Information Processing, vol. 19, pp. 231-252, 2011.

[2] R. V. Yampolskiy and V. Govindaraju, "Behavioural biometrics: a survey and classification," International Journal of Biometrics, vol. 1, no. 1, pp. 81-113, 2008 .

[3] E. Eskin, A. Arnold, M. Prerau, L. Portnoy, and S. Stolfo, "A Geometric Framework for Unsupervised Anomaly Detection," in Applications of Data Mining in Computer Security, pp. 77-101, May 2002.

[4] V. Chandola, A. Banerjee, and V. Kumar, "Anomaly detection: A survey," ACM Computing Surveys (CSUR), vol. 41, no. 3, p. 15, 2009.

[5] T. Fawcett, "ROC graphs: Notes and practical considerations for researchers," Machine Learning, vol. 31, pp. 1-38, 2004.

[6] N. L. Clarke, S. M. Furnell, and P. L. Reynolds, "Biometric authentication for mobile devices," in Proceeding of the 3rd Australian Information Warfare and Security Conference, pp. 61-69, 2002.

[7] H. A. Crawford, A framework for continuous, transparent authentication on mobile devices. PhD thesis, University of Glasgow, Glasgow, Scotland, United Kingdom, 2012.

[8] A. Ross and A. Jain, "Information fusion in biometrics," Pattern recognition letters, vol. 24, no. 13, pp. 2115-2125, 2003.

[9] A. S. Branscomb, "Behaviorally identifying smartphone users," Master's thesis, North Carolina State University, Raleigh, NC, 2013. 
[10] F. Li, N. Clarke, M. Papadaki, and P. Dowland, "Behaviour profiling for transparent authentication for mobile devices," in Proceedings of the 10th European Conference on Information Warfare (ECIW), Tallinn, Estonia, pp. 307-314, 2011.

[11] F. Li, Behaviour Profiling for Mobile Devices. PhD thesis, University of Plymouth, Plymouth, Devon, Great Britain, United Kingdom, 2012.

[12] E. Shi, Y. Niu, M. Jakobsson, and R. Chow, "Implicit authentication through learning user behavior," in Information Security (M. Burmester, G. Tsudik, S. Magliveras, and I. Ilic, eds.), vol. 6531 of Lecture Notes in Computer Science, pp. 99-113, Springer Berlin Heidelberg, 2011.

[13] S. Yazji, R. P. Dick, P. Scheuermann, and G. Trajcevski, "Protecting private data on mobile systems based on spatio-temporal analysis," in Proceedings of the 1st international conference on pervasive and embedded computing and communication systems. SciTePress, pp. 114-123, 2011.

[14] S. Yazji, P. Scheuermann, R. Dick, G. Trajcevski, and R. Jin, "Efficient location aware intrusion detection to protect mobile devices," Personal and Ubiquitous Computing, pp. 1-20, 2013.

[15] S. Yazji, X. Chen, R. Dick, and P. Scheuermann, "Implicit User Reauthentication for Mobile Devices," Lecture Notes in Computer Science: Ubiquitous Intelligence and Computing, vol. 5585, pp. 325-339, 2009.

[16] J. Zhu, P. Wu, X. Wang, and J. Zhang, "Sensec: Mobile security through passive sensing," in Computing, Networking and Communications (ICNC), 2013 International Conference on, pp. 1128-1133, 2013.

[17] K. Wang and S. J. Stolfo, "One-class training for masquerade detection," in 3rd IEEE Conference Data Mining Workshop on Data Mining for Computer Security, 2003.

[18] M. Hall, E. Frank, G. Holmes, B. Pfahringer, P. Reutemann, and I. H. Witten, "The weka data mining software: an update," ACM SIGKDD Explorations Newsletter, vol. 11, no. 1, pp. 10-18, 2009.

[19] N. Eagle, A. S. Pentland, and D. Lazer, "Inferring friendship network structure by using mobile phone data," Proceedings of the National Academy of Sciences, vol. 106, no. 36, pp. 15274-15278, 2009. 
[20] Y. Zheng, X. Xie, and W.-Y. Ma, "GeoLife: A collaborative social networking service among user, location and trajectory," IEEE Data Engineering Bulletin, vol. 33, no. 2, pp. 32-40, 2010.

[21] M. B. Salem and S. J. Stolfo, "Modeling user search behavior for masquerade detection," in Recent Advances in Intrusion Detection, pp. 181-200, Springer, 2011.

[22] A. Madan, M. Cebrian, S. Moturu, K. Farrahi, and S. Pentland, "Sensing the 'health state' of a community," Pervasive Computing, IEEE, vol. 11, no. 4, pp. 36-45, 2012.

[23] M. A. Bayir, M. Demirbas, and N. Eagle, "Discovering spatio-temporal mobility profiles of cellphone users," in World of Wireless, Mobile and Multimedia Networks 65 Workshops, 2009. WoWMoM 2009. IEEE International Symposium on $a$, pp. 1-9, IEEE, 2009.

[24] S. Rawat, Efficient Data Mining Algorithms for Intrusion Detection. PhD thesis, University of Hyderabad, Hyderabad, India, 2005.

[25] A. Jain, K. Nandakumar, and A. Ross, "Score normalization in multimodal biometric systems," Pattern recognition, vol. 38, no. 12, pp. 2270-2285, 2005. 